\title{
SIGMA-COTORSION MODULES OVER VALUATION DOMAINS
}

\author{
SILVANA BAZZONI AND JAN ŠŤOVÍČEK
}

\begin{abstract}
We give a characterization of $\Sigma$-cotorsion modules over valuation domains in terms of descending chain conditions on certain chains of definable subgroups. We prove that pure submodules, direct products and modules elementarily equivalent to a $\Sigma$-cotorsion module are again $\Sigma$-cotorsion. Moreover, we describe the structure of $\Sigma$-cotorsion modules.
\end{abstract}

\section{INTRODUCTION}

The notion of cotorsion abelian groups was introduced independently by Harrison, Nunke and Fuchs in 1959 and 1960. An abelian group is cotorsion if every extension of it by a torsion-free group splits. Matlis and Warfield generalized this notion to modules over arbitrary commutative domains in two different ways, which essentially agree on Dedekind domains. For details on the various notions of cotorsion modules, we refer to [12, VIII.6, XIII.8]. Finally in 1984, Enochs defined cotorsion modules over arbitrary associative rings as the modules $C$ for which $\operatorname{Ext}_{R}^{1}(F, C)=0$ for all flat modules $F$. Over Prüfer domains, Warfield's and Enochs' definitions coincide. Injective and pure injective modules are cotorsion, but the question to characterize the cotorsion modules is hopeless, even in the case of abelian groups. In fact, by $[16]$ the functor $\operatorname{Ext}(\mathbb{Q} / \mathbb{Z},-)$ induces a one-to-one correspondence between the class of reduced torsion groups and the class of adjusted cotorsion groups, that is the class of reduced cotorsion groups containing no non-trivial torsion-free summand. So the classification of adjusted cotorsion groups is equivalent to that of reduced torsion groups.

The notion of $\Sigma$-cotorsion modules is much stronger than the notion of cotorsion: a module $M$ is $\Sigma$-cotorsion if all direct sums of copies of $M$ are cotorsion. The class of $\Sigma$-cotorsion modules contains the class of $\Sigma$-pure injective modules, which have been characterized by Gruson and Jensen [14] and

Date: April 4, 2008.

Key words and phrases. Sigma-cotorsion modules, valuation domains.

First author supported by Università di Padova (Progetto di Ateneo CDPA048343 "Decomposition and tilting theory in modules, derived and cluster categories") and partially supported by grant SAB2005-0139 of the Secretaría de Estado de Univesidades e Investigación del Ministerio de Educación y Ciencia de España within the Research Programme on Discrete and Continuous methods of Ring Theory at the CRM, Barcelona.

Second author supported by the Research Council of Norway through Storforsk-project Homological and geometric methods in algebra, and by GAČR 201/05/H005 and the research project MSM 0021620839.

This paper was finished during the visit of the authors to CRM Barcelona, Spain 2000 Mathematics Subject Classification. Primary: 16D90; 13D07. Secondary: 13G05; $03 \mathrm{E} 75$. 
by Zimmermann [27] in terms of minimum conditions on chains of finitely definable subgroups. This implies in particular that a pure submodule of a $\Sigma$-pure injective module is again pure injective and hence a summand. As a consequence, $\Sigma$-pure injective modules are direct sums of indecomposable modules. There are various classes of rings over which $\Sigma$-pure injective modules have been characterized. Over a Dedekind domain a module is $\Sigma$ pure injective if and only if it is a direct sum of a divisible (hence injective) module and of a module with nonzero annihilator. $\Sigma$-pure injective modules over valuation domains have been classified by Monari-Martinez in [21] and over serial rings by Facchini and Puninski in [11]. For the more general case of commutative Prüfer rings, the structure of $\Sigma$-pure injective modules was given by Camps and Facchini in [6], while a complete classification was obtained in [22] by Prest and Puninski.

The case of $\Sigma$-cotorsion modules is much more complicated. For countable rings, they have been characterized by Guil-Asensio and Herzog in [13] in terms of the descending chain condition on divisibility formulae. For the general case the problem is, however, much harder. Even the case of valuation domains shows that the class of $\Sigma$-cotorsion modules is in general not of countable character in the sense of [13] (see Proposition 4.5). In particular, general $\Sigma$-cotorsion modules cannot be characterized in terms of chain conditions on countable chains of definable subgroups. This reveals a substantial difference from $\Sigma$-pure injective modules.

The problem to characterize $\Sigma$-cotorsion modules over arbitrary rings is still open. One major question, as mentioned by P. Guil-Asensio and I. Herzog [13], is to decide whether the class of $\Sigma$-cotorsion modules is closed under taking pure submodules and elementary equivalent modules. An affirmative answer would build a bridge between model theory and homological algebra, since being $\Sigma$-cotorsion would become a property of certain complete theories of modules instead of single modules. We answer the question in affirmative for valuation domains and, moreover, provide a characterization of $\Sigma$-cotorsion modules from which one can see the necessary divisibility conditions (Theorem 3.8).

The paper is organized as follows. First we note that over a commutative domain, a $\Sigma$-cotorsion reduced module has nonzero annihilator. In particular, this shows that over a Dedekind domain the class of $\Sigma$-cotorsion modules coincides with the class of $\Sigma$-pure injective modules.

In the remainder of the paper our investigation is restricted to the case of valuation domains. An analysis of the relation between vanishing of the Ext functor on direct limits of modules and vanishing of the first derived functor of the inverse limit functor will allow us to give a characterization of $\Sigma$-cotorsion modules $M$ over a valuation domain $R$ in terms of descending chain conditions on submodules of the form $r M$ or $M[r]$, for $r \in R$ (Section 3). Consequently, we can prove that pure submodules, direct products and elementarily equivalent modules of $\Sigma$-cotorsion modules are again $\Sigma$-cotorsion.

In Section 4 we completely characterize divisible $\Sigma$-cotorsion modules. In Section 5 we prove that divisible $\Sigma$-cotorsion modules are building blocks of all $\Sigma$-cotorsion modules in the following sense: an arbitrary $\Sigma$-cotorsion 
module $M$ over a valuation domain $R$ admits a countable filtration by $\Sigma$ cotorsion submodules of the form $I M$, for some ideal $I \leq R$, with the property that the successive factors in the filtration are divisible $\Sigma$-cotorsion over (valuation) factor domains of $R$. Moreover, we characterize $\Sigma$-cotorsion modules this way: a module $M$ is $\Sigma$-cotorsion if and only if the set $\{I M \mid I \leq$ $R\}$ is in fact a countable filtration whose successive factors are $\Sigma$-cotorsion and, by the construction, divisible over suitable valuation factor domains.

\section{Preliminaries}

Recall that an ascending chain $\left(M_{\alpha} \mid \alpha \leq \kappa\right)$ of submodules of a module $M$ indexed by an ordinal number $\kappa$ is called a filtration of $M$ if $M_{0}=0$, $M=M_{\kappa}$ and $M_{\alpha}=\bigcup_{\beta<\alpha} M_{\beta}$ for all limit ordinals $\alpha \leq \kappa$. If $\kappa$ is a regular cardinal, then $\left(M_{\alpha} \mid \alpha \leq \kappa\right)$ is called a $\kappa$-filtration if it is a filtration and, moreover, $M_{\alpha}$ is $<\kappa$-generated for each $\alpha<\kappa$.

When studying classes defined by orthogonality with respect to the Ext functor, such as the class of cotorsion modules, decomposition theorems have been an important tool (see for instance [9, Chapter XII]). We state a general decomposition theorem, valid over an arbitrary ring, which will be crucial in the sequel. Recall that a closed unbouded subset of a regular uncountable cardinal $\kappa$ is a subset that is closed under taking suprema of chains of length $<\kappa$ and is not bounded by any ordinal number smaller than $\kappa$. For more details on set theoretic concepts we refer to [17, Chapter 1].

Theorem 1.1. [24, Theorem 8] Let $R$ be a ring and $M$ an $R$-module. Assume that $\left(M_{\alpha} \mid \alpha \leq \kappa\right)$ is a $\kappa$-filtration of $M$ for some regular uncountable cardinal $\kappa$. Let $\mathcal{B}$ be a class of modules closed under arbitrary direct sums such that $\operatorname{Ext}_{R}^{1}\left(M_{\alpha}, B\right)=0$ for each $\alpha \leq \kappa$ and $B \in \mathcal{B}$. Then there exists a closed unbounded subset $C \subseteq \kappa$ such that $\operatorname{Ext}_{R}^{1}\left(M_{\beta} / M_{\alpha}, B\right)=0$ for each $\alpha, \beta \in C, \alpha<\beta$ and each $B \in \mathcal{B}$.

Let us now recall some basic properties of inverse limits. For an overview of the results in this direction that will be used in this paper, we also refer to [1, Section 1]. Let $R$ be a ring and let $\left(N_{i}, f_{i j}\right)$ be an inverse system of $R$-modules indexed by a directed set $(I, \leq)$. That is, $f_{i j}$ is a homomorphism from $N_{j}$ to $N_{i}, f_{i i}=i d_{N_{i}}$ and $f_{i j} f_{j k}=f_{i k}$ for each $i \leq j \leq k$. The classical result of Jensen [19] says that there is a natural complex

$$
0 \rightarrow \lim _{i \in I} N_{i} \rightarrow \prod_{i_{0} \in I} N_{i_{0}} \stackrel{\Delta^{0}}{\rightarrow} \prod_{i_{0}<i_{1}} N_{i_{0} i_{1}} \stackrel{\Delta^{1}}{\rightarrow} \prod_{i_{0}<i_{1}<i_{2}} N_{i_{0} i_{1} i_{2}} \stackrel{\Delta^{2}}{\rightarrow} \ldots
$$

where $N_{i_{0} i_{1} \ldots i_{n}}=N_{i_{0}}$ for all $i_{0}<i_{1}<\cdots<i_{n}$ in $I$ and

$$
\begin{aligned}
\Delta^{0}\left(\left(x_{i}\right)_{i}\right) & =\left(x_{i}-f_{i j}\left(x_{j}\right)\right)_{i<j} \\
\Delta^{1}\left(\left(x_{i j}\right)_{i<j}\right) & =\left(x_{i k}-x_{i j}-f_{i j}\left(x_{j k}\right)\right)_{i<j<k}
\end{aligned}
$$

Moreover, the first three terms of the complex form an exact sequence; that is $\lim _{i \in I} N_{i}=\operatorname{Ker} \Delta_{0}$. We will use this representation of an inverse limit for computation.

The rest of the complex is, however, not exact in general. This is very closely related to the well-known fact that lim is not an exact functor-it is left exact, but an inverse limit of epimorphisms is not an epimorphism in 
general. More precisely, if we define the $n$-th derived functor of inverse limit as $\lim _{i \in I}^{n} N_{i}=\operatorname{Ker} \Delta^{n} / \operatorname{Im} \Delta^{n-1}$ for $n \geq 1$, we get the following canonical long exact sequence for any inverse system of short exact sequences $0 \rightarrow$ $K_{i} \rightarrow L_{i} \rightarrow N_{i} \rightarrow 0$ indexed by $I$ :

$$
0 \rightarrow \lim _{\longleftarrow} K_{i} \rightarrow \lim _{\longleftarrow} L_{i} \rightarrow \lim _{\longleftarrow} N_{i} \rightarrow \underset{\lim ^{1} K_{i} \rightarrow \lim ^{1} L_{i} \rightarrow \lim ^{1} N_{i} \rightarrow \ldots}{\lim ^{n} K_{i} \rightarrow \lim ^{n} L_{i} \rightarrow \lim ^{n} N_{i} \rightarrow \ldots}
$$

Of special interest are countable inverse systems indexed by natural numbers, that is, the systems of the form

$$
N_{0} \stackrel{f_{0}}{\longleftarrow} N_{1} \stackrel{f_{1}}{\longleftarrow} N_{2} \stackrel{f_{2}}{\longleftarrow} N_{3} \stackrel{f_{3}}{\longleftarrow} \ldots
$$

Here, we write just $f_{i}$ instead of $f_{i, i+1}$. In this case, $\lim ^{1}$ can be computed more easily - there is the following exact sequence:

$$
0 \rightarrow \lim _{\longleftarrow} N_{i} \rightarrow \prod_{i<\omega} N_{i} \stackrel{\tilde{\Delta}}{\rightarrow} \prod_{i<\omega} N_{i} \rightarrow \lim ^{1} N_{i} \rightarrow 0,
$$

where $\tilde{\Delta}\left(\left(x_{i}\right)_{i<\omega}\right)=\left(x_{i}-f_{i}\left(x_{i+1}\right)\right)_{i<\omega}$ for any $\left(x_{i}\right)_{i<\omega} \in \prod_{i<\omega} N_{i}$, see $[25$, $3.5]$.

There is an important connection between vanishing of $\operatorname{Ext}_{R}^{1}\left(\lim _{\longrightarrow} C_{i}, M\right)$ and $\lim ^{1} \operatorname{Hom}_{R}\left(C_{i}, M\right)$ for a countable direct system $\left(C_{i}, g_{i}\right)$ :

Lemma 1.2. Let

$$
C_{0} \stackrel{g_{0}}{\rightarrow} C_{1} \stackrel{g_{1}}{\rightarrow} C_{2} \stackrel{g_{2}}{\rightarrow} C_{3} \stackrel{g_{3}}{\rightarrow} \ldots
$$

be a countable direct system of $R$-modules and let $M$ be any $R$-module. Then the following hold:

(1) If $\operatorname{Ext}_{R}^{1}\left(\underset{\lim }{\longrightarrow} C_{i}, M\right)=0$, then also $\lim ^{1} \operatorname{Hom}_{R}\left(C_{i}, M\right)=0$.

(2) If $\operatorname{Ext}_{R}^{1}\left(C_{i}, M\right)=0$ for each $i<\omega$, then $\operatorname{Ext}_{R}^{1}\left(\lim _{i} C_{i}, M\right) \cong \lim ^{1} \operatorname{Hom}_{R}\left(C_{i}, M\right)$.

Proof. The proof here is in fact a part of the proof of [3, Theorem 5.1]. It is well known that for the countable direct limit above, there is an exact sequence

$$
0 \rightarrow \bigoplus_{i<\omega} C_{i} \stackrel{\phi}{\rightarrow} \bigoplus_{i<\omega} C_{i} \rightarrow \underline{\lim _{\longrightarrow}} C_{i} \rightarrow 0
$$

where, for every $i<\omega, \phi \varepsilon_{i}=\varepsilon_{i}-\varepsilon_{i+1} g_{i}$ and $\varepsilon_{i}: C_{i} \rightarrow \bigoplus_{i<\omega} C_{i}$ denotes the canonical inclusion. When applying the functor $\operatorname{Hom}_{R}(-, M)$ to this sequence, we obtain the following commutative diagram with exact rows and isomorphisms in columns:

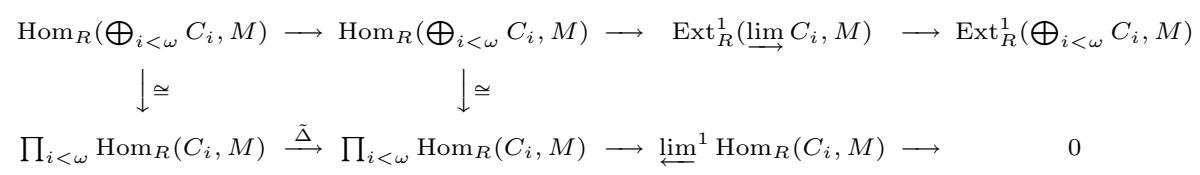

where the map $\tilde{\Delta}$ is relative to the inverse system $\left(\operatorname{Hom}_{R}\left(C_{i}, M\right) ; \operatorname{Hom}_{R}\left(f_{i}, M\right)\right)_{i<\omega}$. Now, if $\operatorname{Ext}_{R}^{1}\left(\lim _{i} C_{i}, M\right)=0$, then $\tilde{\Delta}$ is an epimorphism and (1) follows. If, on the other hand, $\operatorname{Ext}_{R}^{1}\left(C_{i}, M\right)=0$ for each $i<\omega$, then $\lim ^{1} \operatorname{Hom}_{R}\left(C_{i}, M\right) \cong$ $\operatorname{Ext}_{R}^{1}\left(\lim _{\longrightarrow} C_{i}, M\right)$ and (2) follows. 
The main problem with this approach is that it is difficult in general to determine whether $\lim ^{1}$ vanishes for a given inverse system. For countable inverse systems, however, this is tractable. To this end, we recall a notion introduced by Grotendieck in [15, 13.1.1]. A countable inverse system $\left(N_{i}\right)_{i<\omega}$ satisfies the Mittag-Leffler condition, if for every natural number $i$, the chain of modules

$$
N_{i} \geq f_{i}\left(N_{i+1}\right) \geq f_{i, i+2}\left(N_{i+2}\right) \geq \cdots \geq f_{i, i+k}\left(N_{i+k}\right) \geq \cdots
$$

is stationary. If $\left(N_{i}\right)_{i<\omega}$ is an inverse system, let us denote by $\left(N_{i}^{(\omega)}\right)_{i<\omega}$ the system whose $i$-th term consists of the countable direct sum $N_{i}^{(\omega)}$ of copies of $N_{i}$ and whose maps are the obvious coproduct maps. There is a connection between the Mittag-Leffler condition and vanishing of $\lim ^{1} N_{i}$ and $\lim ^{1} N_{i}^{(\omega)}$, the origins of which could be traced back to [15] and [10]:

Theorem 1.3. Let $R$ be a ring and let

$$
N_{0} \stackrel{f_{0}}{\longleftarrow} N_{1} \stackrel{f_{1}}{\longleftarrow} N_{2} \stackrel{f_{2}}{\longleftarrow} N_{3} \stackrel{f_{3}}{\longleftarrow} \ldots
$$

be a countable inverse system of $R$-modules. Then the following hold:

(1) [25, Proposition 3.5.7] If $\left(N_{i}\right)_{i<\omega}$ satisfies the Mittag-Leffler condition, then $\lim ^{1} N_{i}=0$.

(2) [1, Theorem 1.3] $\left(N_{i}\right)_{i<\omega}$ satisfies the Mittag-Leffler condition if and only if $\lim ^{1} N_{i}^{(\omega)}=0$.

When combining the results above, we get the following useful corollary:

Corollary 1.4. [3, Theorem 5.1] [1, Theorem 1.3] Let $R$ be a ring and let $\left(C_{i}, g_{i}\right)$ be a countable direct system of finitely generated $R$-modules. Let $M$ be a module such that $\operatorname{Ext}_{R}^{1}\left(C_{i}, M^{(X)}\right)=0$ for each $i<\omega$ and each set $X$. Then the following are equivalent:

(1) $\operatorname{Ext}_{R}^{1}\left(\stackrel{\lim }{\longrightarrow} C_{i}, M^{(X)}\right)=0$ for any set $X$.

(2) $\operatorname{Ext}_{R}^{1}\left(\overleftrightarrow{\lim _{i}} C_{i}, M^{(\omega)}\right)=0$.

(3) The inverse system $\left(\operatorname{Hom}_{R}\left(C_{i}, M\right), \operatorname{Hom}_{R}\left(g_{i}, M\right)\right)$ satisfies the MittagLeffler condition.

We will also be interested in larger inverse systems indexed by well-ordered sets. Let $\kappa$ be an ordinal number and $\left(N_{\alpha}, f_{\alpha \beta}\right)_{\alpha<\beta<\kappa}$ be an inverse system indexed by $\kappa$. We call such a system continuous if, for every limit ordinal $\beta<\kappa, N_{\beta}=\lim _{\alpha<\beta} N_{\alpha}$. A continuous inverse system $\left(N_{\alpha}, f_{\alpha \beta}\right)_{\alpha<\beta<\kappa}$ is called weakly flabby if there is $\delta<\kappa$ such that $f_{\alpha \beta}$ is an epimorphism whenever $\alpha, \beta<\kappa$ and $\delta<\alpha<\beta$. It is not difficult to obtain the following result using transfinite induction:

Theorem 1.5. [19, Theorem 1.8] Let $R$ be a ring. Let $\kappa$ be an ordinal number and $\left(N_{\alpha}, f_{\alpha \beta}\right)_{\alpha<\beta<\kappa}$ be a continuous weakly flabby inverse system of $R$-modules. Then $\lim ^{1} N_{\alpha}=0$.

Before entering in the discussion of $\Sigma$-cotorsion modules over valuation domains, we illustrate a consequence of Corollary 1.4 (see also [13, Theorem $12])$. 
Definition 1.6. We say that an $R$-module $M$ is $\aleph_{0}$-cotorsion (in the sense of [13]) if $M^{\left(\aleph_{0}\right)}$ is cotorsion.

Lemma 1.7. Let $R$ be a ring and $M$ an $\aleph_{0}$-cotorsion left $R$-module. If $R t_{0} \geq R t_{1} \geq \cdots \geq R t_{n} \geq \ldots$ is a descending chain of left ideals of $R$, then the descending chain $t_{0} M \geq t_{1} M \geq \cdots \geq t_{n} M \geq \ldots$ of subgroups of $M$ is stationary.

Proof. Let $s_{0}=t_{0}$ and for every $n \geq 1$, let $s_{n} \in R$ be such that $t_{n}=s_{n} t_{n-1}$. Consider the countable direct system $\left(R_{n}, g_{n}\right), n \geq 0$ where $R_{n}=R$, for every $n \geq 0$ and $g_{n}$ is the right multiplication by $s_{n}$. The direct limit of the direct system $\left(R_{n}, g_{n}\right)$ is a countably presented flat module, hence the assumption on $M$ and Corollary 1.4 give us that the inverse system of abelian groups $\left(\operatorname{Hom}_{R}\left(R_{i}, M\right), \operatorname{Hom}_{R}\left(g_{i}, M\right)\right)$ satisfies the Mittag-Leffler condition. This implies, in particular, that the chain $t_{0} M \geq t_{1} M \geq \cdots \geq t_{n} M \geq \ldots$ of subgroups of $M$ is stationary.

Recall that a module over a commutative domain is called bounded if it has nonzero annihilator. An application of the preceding result to commutative domains yields:

Proposition 1.8. Let $R$ be a commutative domain. The following hold:

(1) An $\aleph_{0}$-cotorsion reduced $R$-module is bounded.

(2) If $R$ is a Dedekind domain, then an $R$-module is $\aleph_{0}$-cotorsion if and only if it is a direct sum of a divisible module and a bounded module. In particular, the classes of $\aleph_{0}$-cotorsion, $\Sigma$-cotorsion and $\Sigma$-pure injective modules coincide.

Proof. (1) Let $M$ be an $\aleph_{0}$-cotorsion reduced $R$-module. If $M$ is not bounded, then using the hypothesis that $M$ has no nonzero divisible submodules, we can construct a chain of principal left ideals $R t_{0}>R t_{1}>\cdots>R t_{n}>\ldots$ and a strictly decreasing chain of submodules of $M$ of the form $t_{0} M>$ $t_{1} M>\cdots>t_{n} M>\ldots$, contradicting Lemma 1.7.

(2) It is well known that over a Dedekind domain, a direct sum of a divisible module and a bounded module is $\Sigma$-pure injective, hence also $\Sigma$ cotorsion. Conversely, if $M$ is $\aleph_{0}$-cotorsion and $D$ is the maximal divisible submodule of $M$, then $D$ is injective, hence $M=D \oplus M / D$. Now $M / D$ is a reduced $\aleph_{0}$-cotorsion module, hence by part (1), $M / D$ is bounded. This shows that, over Dedekind domains, the classes of $\aleph_{0}$-cotorsion, $\Sigma$-cotorsion and $\Sigma$-pure-injective modules coincide.

We will also need to decide, in particular situations, when a divisible module over a commutative domain is $\Sigma$-cotorsion. For example, this is clearly always the case for Dedekind domains, but we will see later that there are domains where such an implication does not hold. However, as the following proposition shows, they must be all uncountable:

Proposition 1.9. Any divisible module over a countable commutative domain is $\Sigma$-cotorsion.

Proof. By [13, Theorems 11 and 12], a module over a countable ring $S$ is $\Sigma$ cotorsion if and only if $\operatorname{Ext}_{S}^{1}\left(F, M^{(X)}\right)=0$ for every countably presented flat 
$S$-module $F$ and every set $X$. Every countably presented flat module $F$ is a countable direct limit of finitely generated projective (or even free) modules (see [23]); hence $F$ has projective dimension at most one, by [19]. Now, by $[4$, Corollary 8.2$]$, for any commutative domain $R$, the class of divisible $R$-modules is the right Ext-orthogonal to the class of modules of projective dimension at most one, that is $\operatorname{Ext}_{R}^{1}(N, D)=0$, for every divisible module $D$ and for every module $N$ of projective dimension at most one. The claim follows since direct sums of divisible modules are divisible.

Recall that a commutative domain $R$ is called a valuation domain if its ideals form a chain with respect to inclusion. For proofs of the following facts, unexplained terminology, and other results on modules over valuation domains we refer to [12, XIII].

Let $R$ be a valuation domain. Then the class of flat modules coincides with the class of torsion-free modules. This follows from the fact that the weak global dimension of any valuation domain is at most 1 and, subsequently, the class of flat modules is closed under taking submodules. As a consequence, cotorsion modules have injective dimension at most one and an $R$-module $M$ is cotorsion if and only if $\operatorname{Ext}_{R}^{1}(J, M)=0$ for every submodule $J \leq Q$ where $Q$ is the quotient field of $R$ (see [12, XIII.8]). Hence, a module $M$ over a valuation domain is $\Sigma$-cotorsion if and only if $\operatorname{Ext}_{R}^{1}\left(J, M^{(X)}\right)=0$ for $J \leq Q$ and every set $X$. If moreover, there is an infinite cardinal $\kappa$ such that every submodule $J \leq Q$ is $\leq \kappa$-presented, then a module $M$ is $\Sigma$-cotorsion if and only if $\operatorname{Ext}_{R}^{1}\left(J, M^{(\kappa)}\right)=0$ for $J \leq Q$.

Let $P$ be a prime ideal of $R$. If $P$ is maximal, it is well known that $P$ is either idempotent or principal. If $P$ is not maximal, we can pass to the corresponding localization: $P$ is either idempotent or principal in $R_{P}$. Note that for valuation domains, $P$ and $P \cdot R_{P}$ coincide when viewed as subsets of the quotient field (see [12, II.4]).

If $R$ is a valuation domain and $M$ is a nonzero $R$-module, there are two prime ideals associated to $M$ as follows:

$$
M^{\#}=\{r \in R \mid r M<M\} ; M_{\#}=\{r \in R \mid \exists 0 \neq x \in M, r x=0\}
$$

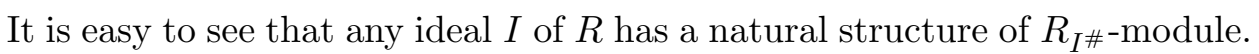
If $M$ is an $R$-module and $r, s \in R$, we let

$$
M[s]=\{x \in M \mid s x=0\}
$$

and

$$
r^{-1} M[s]=\{x \in M \mid r x \in M[s]\} .
$$

The following lemma has a straightforward proof:

Lemma 1.10. Let $R$ be a valuation domain and $M$ be a nonzero $R$-module. Let $r, s \in R$ and $r M \neq 0$. Then the following hold:

(1) $r s M<r M$ if and only if $s M+M[r]<M$ if and only if $s \in(r M)^{\#}$.

(2) $M[r s]>M[r]$ if and only if $(r M)[s]>0$ if and only if $s \in(r M)_{\#}$.

(3) $M[r s]=r^{-1} M[s]$. 


\section{Ext-orthogonality to Countable submodules of $Q$}

From now on $R$ will always be a valuation domain and all modules will be $R$-modules. We denote by $Q$ the quotient field of $R$. First, we will look at the modules $M$ that $\operatorname{Ext}_{R}^{1}\left(J, M^{(X)}\right)=0$ for every countably generated submodule $J \leq Q$ and any set $X$. The following proposition gives a more convenient characterization:

Proposition 2.1. Let $M$ be an $R$-module. Then the following conditions are equivalent:

(1) $\operatorname{Ext}_{R}^{1}\left(J, M^{(X)}\right)=0$ for every countably generated $R$-submodule $J \leq$ $Q$ and every set $X$.

(2) The set $\{t M \mid t \in R\}$ is well-ordered with respect to inclusion.

Proof. Assume (1) and denote $\mathcal{S}=\{t M \mid t \in R\}$. Since $R$ is a valuation domain, $\mathcal{S}$ is totally ordered with respect to inclusion. Now, let

$$
t_{0} M \geq t_{1} M \geq t_{2} M \geq \ldots
$$

be a descending chain in $\mathcal{S}$. We can always assume that $t_{i}$ divides $t_{i+1}$ for each $i$. If this is not the case, we can construct by induction a new sequence $\left(t_{i}^{\prime}\right)_{i<\omega}$ of elements of $R$ such that $t_{i}^{\prime}$ divides $t_{i+1}^{\prime}$ and $t_{i} M=t_{i}^{\prime} M$ for each $i$ : Let $t_{0}^{\prime}=t_{0}$. Assume $t_{i}^{\prime}$ has already been constructed. Then either $t_{i}^{\prime} M=t_{i} M>t_{i+1} M$ or $t_{i}^{\prime} M=t_{i+1} M$. In the first case $t_{i}^{\prime}$ divides $t_{i+1}$ since $t_{i+1}$ cannot divide $t_{i}^{\prime}$, and we can put $t_{i+1}^{\prime}=t_{i+1}$. In the second case we can simply put $t_{i+1}^{\prime}=t_{i}^{\prime}$. Hence we have a countable direct system

$$
R \stackrel{t_{0}}{\longrightarrow} R \stackrel{t_{1} t_{0}^{-1}}{\longrightarrow} R \stackrel{t_{2} t_{1}^{-1}}{\longrightarrow} R \stackrel{t_{3} t_{2}^{-1}}{\longrightarrow} \ldots
$$

Let $J$ be the direct limit of this system. Then it is straightforward to see that $J$ is isomorphic to the submodule of $Q$ generated by the set $\left\{t_{i}^{-1} \mid i<\right.$ $\omega\}$. By assumption, $\operatorname{Ext}_{R}^{1}\left(J, M^{(\omega)}\right)=0$. Therefore, by Corollary 1.4, the inverse system

$$
M \stackrel{t_{0}}{\longleftarrow} M \stackrel{t_{1} t_{0}^{-1}}{\longleftarrow} M \stackrel{t_{2} t_{1}^{-1}}{\longleftarrow} M \stackrel{t_{3} t_{2}^{-1}}{\longleftarrow} \ldots
$$

satisfies the Mittag-Leffler condition. In particular this means that the chain $t_{0} M \geq t_{1} M \geq t_{2} M \geq \ldots$ stops. Hence, since $\mathcal{S}$ is totally ordered and the chain has been chosen arbitrarily, $\mathcal{S}$ is well-ordered.

Conversely, if $\mathcal{S}$ is well-ordered, then every countable inverse system of the form

$$
M \stackrel{r_{0}}{\longleftarrow} M \stackrel{r_{1}}{\longleftarrow} M \stackrel{r_{2}}{\longleftarrow} \ldots
$$

clearly satisfies the Mittag-Leffler condition. And every countably generated submodule $J \leq Q$ is generated by some set of elements $\left\{x_{i} \mid i<\omega\right\}$ of $Q$ such that $x_{i} x_{i+1}^{-1} \in R$. If we denote $r_{i}=x_{i} x_{i+1}^{-1}$, we get $\operatorname{Ext}_{R}^{1}\left(J, M^{(X)}\right)=0$ for every set $X$ by Corollary 1.4. Hence, $M$ satisfies (1).

In the sequel, we will will give several consequences of condition 2 from Proposition 2.1. First, we start with a relation between the ideals $M_{\#}$ and $M^{\#}$, defined in Section 1.

Lemma 2.2. Let $M$ be a nonzero $R$-module such that $\{t M \mid t \in R\}$ is well-ordered with respect to inclusion. Then: 
(1) $M_{\#} \geq M^{\#}$. In particular $M$ is naturally an $R_{M_{\#}}$-module.

(2) $s M<t M$ implies $M[s]>M[t]$ for any $s, t \in R$.

Proof. (1). Assume that there is $s \in M^{\#} \backslash M_{\#}$. Then, for every $n \geq 1$, $s^{n} \notin M_{\#}$; hence $M\left[s^{n}\right]=0$. Applying Lemma 1.10 (1), it is easy to show that $s^{n} M$ is a strictly descending chain of submodules of $M$. This contradicts the assumption on $M$.

The second statement in (1) follows by noting that, if $M_{\#} \geq M^{\#}$, then the multiplication by each element $r \notin M_{\#}$ induces an isomorphism of $M$.

(2) follows easily from (1) by Lemma 1.10.

If we fix a module $M$, a general fact about applying $(-)^{\#}$ and $(-)_{\#}$ to the elements of the set $\mathcal{S}=\{t M \mid t \in R\}$ is that we get an order-preserving map from $\mathcal{S} \backslash\{0\}$ to $\operatorname{Spec}(R)$. We state this as a lemma.

Lemma 2.3. Let $M$ be a nonzero $R$-module and $s_{1}, s_{2} \in R$ such that $0<$ $s_{1} M \leq s_{2} M$. Then $\left(s_{1} M\right)^{\#} \leq\left(s_{2} M\right)^{\#}$ and $\left(s_{1} M\right)_{\#} \leq\left(s_{2} M\right)_{\#}$.

Proof. The conclusion is clear when $s_{1} M=s_{2} M$. Let us then assume that $s_{1} M<s_{2} M$ and thus $s_{1} R<s_{2} R$. The inclusion $\left(s_{1} M\right)_{\#} \leq\left(s_{2} M\right)_{\#}$ follows immediately from the definition. By Lemma 1.10, $r \in\left(s_{i} M\right)^{\#}$ if and only if $r M+M\left[s_{i}\right]<M$ for $i=1,2$. But by assumption, we have $M\left[s_{1}\right] \geq M\left[s_{2}\right]$, hence $r \in\left(s_{1} M\right)^{\#}$ implies $r \in\left(s_{2} M\right)^{\#}$.

Next, if $M$ is a nonzero module such that $\mathcal{S}=\{t M \mid t \in R\}$ is wellordered, there is a minimal nonzero element of $\mathcal{S}$, say $s M$. Note that by the choice, $r s M$ is either 0 or $s M$ for any $r \in R$; therefore the annihilitor $\operatorname{Ann}_{R}(s M)$ is equal to $(s M)^{\#}$. The following lemma gives a useful connection between $(s M)^{\#}=\operatorname{Ann}_{R}(s M)$ and $\operatorname{Ann}_{R}(M)$ :

Lemma 2.4. Let $M$ be a nonzero bounded module with annihilator $A$ and assume that the set $\mathcal{S}=\{t M \mid t \in R\}$ is well-ordered with respect to inclusion. Let $s M$ be the minimum nonzero element in $\mathcal{S}$ and let $L_{0}=(s M)^{\#}$. The following hold:

(1) $A$ is an $R_{L_{0}}$-module;

(2) $A=L_{0}^{m}$, for some $m \geq 1$ and if $m>1$, then $L_{0}$ is a principal ideal of $R_{L_{0}}$.

(3) $A=s L_{0}$ and $L_{0}=\sqrt{A}$.

Proof. (1) We show that $A^{\#} \leq L_{0}$. Let $r \notin L_{0}$, then $r s M=s M$. Hence $\operatorname{Ann}_{R}(s M)=s^{-1} A=\operatorname{Ann}_{R}(r s M)=(r s)^{-1} A$. So $r^{-1} A=A$ and thus

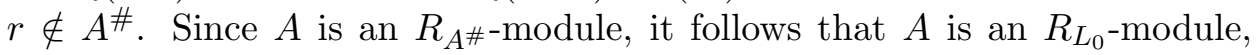
too.

(2) Clearly $A \leq L_{0}$. Consider the ring $R_{L_{0}} / A$; it is a local uniserial ring with maximal ideal $L_{0} / A$. We claim that it is an artinian ring. Assume for the moment that this is not the case. Then it is easy to see, using the uniseriality, that $R_{L_{0}} / A$ is not even perfect. That is, there exists a strictly decreasing chain

$$
t_{1} R_{L_{0}}>t_{1} t_{2} R_{L_{0}}>\cdots>t_{1} t_{2} \cdots t_{n} R_{L_{0}}>\cdots
$$

such that $t_{n} \in L_{0}$ and $t_{1} t_{2} \ldots t_{n} \notin A$ for any natural number $n$. By the minimality of $L_{0}$ in the set $\left\{(t M)^{\#} \mid t \in R \backslash A\right\}$, this implies that the 
chain $t_{1} M>t_{1} t_{2} M>\cdots>t_{1} t_{2} \cdots t_{n} M>\ldots$ is strictly decreasing-a contradition to our assumption. Hence, the claim is proved.

Therefore, the maximal ideal $L_{0} / A$ is nilpotent, that is, there is an index $n$ such that $L_{0}^{n} \leq A$. Now, every prime ideal $L$ of the valuation domain $R$ is either idempotent or a principal ideal of the localization $R_{L}$. Thus, either $L_{0}=A$ or $L_{0}=b R_{L_{0}}$, for some $b \in L_{0}$. The ideals of $R_{L_{0}}$ containing a power of the maximal ideal are necessarily powers of the maximal ideal. Thus by part (1), $A=L_{0}^{m}$ for some integer $m \geq 1$.

(3) Since $s M$ is the minimum nonzero element in $\mathcal{S}$, we have that for every $r \in R$ either $r s M=0$ or $r s M=s M$. Hence $L_{0}=\operatorname{Ann}(s M)$. But $\operatorname{Ann}(s M)=s^{-1} A$, hence $A=s L_{0}$. The other equality follows immediately by (2) and the definition of the radical of an ideal.

As the last statement in this section, we seek to restrict the set of submodules of $Q$ to which $M^{(X)}$ has to be Ext-orthogonal in order that $M$ be $\Sigma$-cotorsion. Before doing this, we will prove a lemma that will be also useful later.

Lemma 2.5. Let $M$ be a nonzero $R$-module and $P$ be a prime ideal of $R$. Then the following hold:

(1) If $P \leq \operatorname{Ann}_{R}(M)$, then $M$ is $\Sigma$-cotorsion as an $R$-module if and only if it is $\Sigma$-cotorsion as an $(R / P)$-module.

(2) If $P \geq M_{\#}$, then $M$ is $\Sigma$-cotorsion as an $R$-module if and only if it is $\Sigma$-cotorsion as an $R_{P}$-module.

Proof. (1). For every $(R / P)$-module $M$ and every $R$-module $Y$, we have the following homological formula (see [7, VI, 4.1.3]):

$$
\operatorname{Ext}_{R / P}^{1}\left(R / P \otimes_{R} Y, M\right) \cong \operatorname{Ext}_{R}^{1}(Y, M),
$$

provided that $\operatorname{Tor}_{n}^{R}(R / P, Y)=0$ for every $n$. Thus if $Y$ is a (torsion-free) $R$-submodule of $Q$, the formula gives the isomorphism

$$
\operatorname{Ext}_{R / P}^{1}\left(Y / P Y, M^{(X)}\right) \cong \operatorname{Ext}_{R}^{1}\left(Y, M^{(X)}\right) .
$$

Since $P$ is a prime ideal, it is easy to check that $Y / P Y$ is a torsion-free $(R / P)$-module. Hence, if $M$ is a $\Sigma$-cotorsion $(R / P)$-module, then $\operatorname{Ext}_{R}^{1}\left(Y, M^{(X)}\right)=$ 0 for every set $X$, and $M$ is a $\Sigma$-cotorsion $R$-module.

Conversely, if $J$ is a nonzero $R / P$-submodule of the quotient field $R_{P} / P$ of $R / P$, then $J=Y / P$ for some $P<Y \leq R_{P}$ and thus $P Y=P$. Therefore $\operatorname{Ext}_{R}^{1}\left(Y, M^{(X)}\right)=0$ implies that $\operatorname{Ext}_{R / P}^{1}\left(Y / P, M^{(X)}\right)=0$ for every set $X$ and, subsequently, $M$ is a $\Sigma$-cotorsion $(R / P)$-module.

(2). If $P \geq M_{\#}$ and $M$ is a $\Sigma$-cotorsion $R$-module, then also $P \geq M^{\#}$ by Proposition 2.1 and Lemma 2.2. Hence, as in Lemma 2.2 (1), multiplying by every $r \in R \backslash P$ induces an automorphism of $M$ and $M$ is naturally an $R_{P}$-module. Moreover, using the well known homological formula

$$
\operatorname{Ext}_{R}^{1}(A, B) \cong \operatorname{Ext}_{R_{P}}^{1}\left(R_{P} \otimes_{R} A, B\right),
$$

(see [7, VI, 4.1.3] and use the fact that $R_{P}$ is a flat $R$-module), we infer that $M$ is $\Sigma$-cotorsion as an $R_{P}$-module.

On the other hand, if $M$ is an $R_{P}$-module and it is $\Sigma$-cotorsion as an $R_{P}$-module, then we have $\operatorname{Ext}_{R}^{1}\left(J, M^{(X)}\right) \cong \operatorname{Ext}_{R_{P}}^{1}\left(R_{P} \otimes_{R} J, M^{(X)}\right)=0$ 
for any $R$-submodule $J \leq Q$ and any set $X$. Hence $M$ is a $\Sigma$-cotorsion $R$-module.

Now we state the condition saying which submodules of $Q$ have to be tested to see that a given $R$-module $M$ such that $\{t M \mid t \in R\}$ is wellordered is $\Sigma$-cotorsion.

Proposition 2.6. Let $M$ be a nonzero module such that $\mathcal{S}=\{t M \mid t \in R\}$ is well-ordered. Denote $L_{0}=\sqrt{\operatorname{Ann}_{R}(M)}$ and $P=M_{\#}$. Then the following conditions are equivalent:

(1) $M$ is a $\Sigma$-cotorsion $R$-module,

(2) $\operatorname{Ext}_{R}^{1}\left(J, M^{(X)}\right)=0$ for every set $X$ and for every $R_{P}$-submodule $J$ of $Q$ such that $L_{0}<J \leq R_{L_{0}}$.

Proof. (1) $\Longrightarrow(2)$. This is clear, since $\operatorname{Ext}_{R}^{1}\left(J, M^{(X)}\right)=0$ even for any $R$-submodule $J \leq Q$ and any set $X$ provided $M$ is $\Sigma$-cotorsion.

$(2) \Longrightarrow(1)$. Assume that $(2)$ holds. First note that $L_{0}$ is a prime

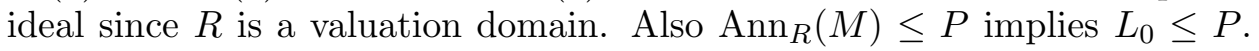
Moreover, by Lemma $2.5(2)$, we can assume that $P$ is the maximal ideal of $R$.

Next assume that $H$ is some prime ideal contained in $\operatorname{Ann}_{R}(M)$. By Lemma 2.5 (1), $M$ is $\Sigma$-cotorsion as an $R$-module if and only if $M$ is $\Sigma$ cotorsion as an $R / H$-module. Also by the proof of Lemma 2.5 (1), the latter condition is equivalent to $\operatorname{Ext}_{R}^{1}\left(J, M^{(X)}\right)=0$ for every $R$-submodule $J \leq Q$ such that $H<J \leq R_{H}$, since we have to test $\operatorname{Ext}_{R / H}\left(J / H, M^{(X)}\right)=0$ only for nonzero $(R / H)$-submodules $J / H$ of the quotient field $R_{H} / H$.

By Lemma $2.4(2)$, we know that $\operatorname{Ann}_{R}(M)=L_{0}^{m}$ for some positive integer $m$. If $m=1$, we can take $H=L_{0}$ and the proof is finished by the preceding paragraph. Assume that $\operatorname{Ann}_{R}(M)=L_{0}^{m}$ for some $m>1$. Then $L_{0}=b R_{L_{0}}$ for some $b \in L_{0}$. Put

$$
H=\bigcap_{i<\omega} L_{0}^{i}
$$

It follows that $H$ is a prime ideal contained in the annihilator $\operatorname{Ann}_{R}(M)$ and $R_{H}=\bigcup_{i<\omega} b^{-i} R_{L_{0}}$. Note that the chain $\left(b^{-i} R_{L_{0}}\right)_{i<\omega}$ viewed as a countable direct system is isomorphic to the system:

$$
R_{L_{0}} \stackrel{b \cdot}{\rightarrow} R_{L_{0}} \stackrel{b \cdot}{\rightarrow} R_{L_{0}} \stackrel{b \cdot}{\rightarrow} \ldots
$$

Hence, if we apply the functor $\operatorname{Hom}_{R}\left(-, M^{(X)}\right)$ to it, the resulting inverse system $\left(\operatorname{Hom}_{R}\left(b^{-i} R_{L_{0}}, M^{(X)}\right)\right)_{i<\omega}$ clearly satisfies the Mittag-Leffler condition since $b^{m} M=0$. It follows that $\varliminf_{i<\omega}^{1} \operatorname{Hom}_{R}\left(b^{-i} R_{L_{0}}, M^{(X)}\right)=0$ by Theorem 1.3. Consequently, since $\operatorname{Ext}_{R}^{1}\left(R_{L_{0}}, M^{(X)}\right)=0$ by assumption, $\operatorname{Ext}_{R}^{1}\left(R_{H}, M^{(X)}\right)=0$ for every set $X$ by Lemma $1.2(2)$.

Hence, to see that $M$ is a $\Sigma$-cotorsion $R$-module, we only have to prove that $\operatorname{Ext}_{R}^{1}\left(J, M^{(X)}\right)=0$ for every set $X$ and every $J \leq Q$ such that $H<$ $J<R_{H}$. For this, it is enough to show that for every such $J$ there is $K \cong J$ such that $L_{0}<K \leq R_{L_{0}}$. If $J$ is already between $L_{0}$ and $R_{L_{0}}$, we can just take $K=J$. If not, assume first that $H<J \leq L_{0}$. Then there is a (unique) $i \geq 1$ such that $L_{0}^{i+1}<J \leq L_{0}^{i}$. Therefore, taking $K=b^{-i} J$ gets us to the 
wanted range. Finally, if $R_{L_{0}}<J<R_{H}$, we can similarly take $K=b^{i} J$ for a suitable $i \geq 1$.

\section{Characterization By Chain CONDitions}

In this section again, $R$ will always be a valuation domain and $Q$ its quotient field. We characterize $\Sigma$-cotorsion $R$-modules by certain descending chain conditions on definable subgroups. The whole result is summarized in Theorem 3.8. This characterization has an important consequence-for valuation domains it answers in affirmative the question posed by GuilAsensio and Herzog [13] on whether pure submodules of $\Sigma$-cotorsion modules are again cotorsion, see Theorem 3.9.

In the case in which all $R$-submodules of $Q$ are countably generated, such a characterization is already given by Proposition 2.1. For "larger" domains the situation is, however, more complicated. We start with the following definition:

Definition 3.1. Let $\kappa$ be a regular cardinal. Then a $\kappa$-factoring system over $R$ is a family $\mathfrak{F}=\left(r_{\alpha \beta}\right)_{\alpha<\beta<\kappa}$ of nonzero elements of $R$ such that $r_{\alpha \beta} r_{\beta \gamma}=r_{\alpha \gamma}$ for all ordinal numbers $\alpha<\beta<\gamma<\kappa$.

It is easy to see that we have a straightforward correspondence between nonzero $\kappa$-generated submodules of $Q$ and $\kappa$-factoring systems. Namely:

(1) Given a $\kappa$-generated submodule $0 \neq J \leq Q$, we can express $J$ as a union of an ascending well-ordered chain $\left(t_{\gamma} R\right)_{\gamma<\kappa}$ where $0 \neq t_{\gamma} \in Q$ for all $\gamma$. Then clearly $t_{\alpha} t_{\beta}^{-1} \in R$ for all $\alpha<\beta<\kappa$ and the family $\mathfrak{F}=\left(r_{\alpha \beta}\right)_{\alpha<\beta<\kappa}$ given by $r_{\alpha \beta}=t_{\alpha} t_{\beta}^{-1}$ is a $\kappa$-factoring system.

(2) Given a $\kappa$-factoring system $\mathfrak{F}=\left(r_{\alpha \beta}\right)_{\alpha<\beta<\kappa}$, then $J^{\prime}=\bigcup_{\gamma<\kappa} r_{0 \gamma}^{-1} R$ is a (nonzero) $\kappa$-generated submodule of $Q$.

Moreover, if we start with $J \leq Q$ and get a $\kappa$-factoring system $\mathfrak{F}$ as in (1), and we further construct $J^{\prime} \leq Q$ as in (2), then obviously $J \cong J^{\prime}$. Note also that the minimal number of generators of any submodule of $Q$ (and of any uniserial module in general) is a regular cardinal (see [12, II.4]). Next, we consider the aforementioned descending chain conditions.

Definition 3.2. Let $M$ be an $R$-module, $\kappa$ an uncountable regular cardinal and $\mathfrak{F}=\left(r_{\alpha \beta}\right)_{\alpha<\beta<\kappa}$ a $\kappa$-factoring system over $R$. We say that the condition $C C(\mathfrak{F}, M)$ is satisfied if

$C C(\mathfrak{F}, M)$ : There exists a closed unbounded subset $C \subseteq \kappa$ with the property that for each $\gamma \in C$, the descending chain $\left(M\left[r_{\alpha \gamma}\right]\right)_{\alpha<\gamma}$ is stationary.

And for a module $M$ and an uncountable regular cardinal $\kappa$ we say that condition $C C(\kappa, M)$ is satisfied if

$$
\begin{array}{ll}
C C(\kappa, M): & \text { Condition } C C(\mathfrak{F}, M) \text { is satisfied for every } \kappa \text {-factoring } \\
& \text { system } \mathfrak{F} .
\end{array}
$$

We will see that there is a close connection between conditions $C C(\kappa, M)$ and $M$ being $\Sigma$-cotorsion. We start with the following preliminary lemma: 
Lemma 3.3. Let $M$ be an $R$-module satisfying the condition $C C\left(\aleph_{1}, M\right)$. Then there are no strictly descending chains of the form $\left(M\left[t_{\alpha}\right]\right)_{\alpha<\aleph_{1}}, t_{\alpha} \in$ $R$.

Proof. Let $M$ be an $R$-module satisfying the condition $C C\left(\aleph_{1}, M\right)$ and suppose that there are elements $\left(t_{\alpha}\right)_{\alpha<\aleph_{1}}$ in $R$ such that the chain $\left(M\left[t_{\alpha}\right]\right)_{\alpha<\aleph_{1}}$ is strictly descending. Then obviously $t_{\beta}$ properly divides $t_{\alpha}$ for every $\alpha<$ $\beta<\aleph_{1}$. W.l.o.g. $M\left[t_{\alpha}\right]<M$ for every $\alpha<\aleph_{1}$. Put $r_{\alpha \beta}=t_{\alpha} t_{\beta}^{-1}$; then clearly $\mathfrak{F}=\left(r_{\alpha \beta}\right)_{\alpha<\beta<\aleph_{1}}$ is an $\aleph_{1}$-factoring system. By Lemma 1.10, $M\left[t_{\alpha}\right]>M\left[t_{\beta}\right]$ implies $r_{\alpha \beta} \in\left(t_{\beta} M\right)_{\#}$. We have $t_{\beta}=t_{\gamma} r_{\beta \gamma}$; hence $t_{\beta} M \leq r_{\beta \gamma} M$ and also $\left(t_{\beta} M\right)_{\#} \leq\left(r_{\beta \gamma} M\right)_{\#}$ by Lemma 2.3 whenever $\beta<\gamma<\kappa$. Thus, $r_{\alpha \beta} \in\left(r_{\beta \gamma} M\right)_{\#}$ and by Lemma 1.10 we conclude that $M\left[r_{\alpha \gamma}\right]>M\left[r_{\beta \gamma}\right]$, for every $\alpha<\beta<\gamma<\aleph_{1}$. This clearly contradicts the condition $C C(\mathfrak{F}, M)$.

Next, we give a necessary condition for a module $M$ to be Ext-orthogonal to $\leq \kappa$-generated submodules of $Q$ for a given $\kappa$. This in turn implies a necessary condition for a module to be $\Sigma$-cotorsion.

Lemma 3.4. Let $M$ an $R$-module and $\kappa$ an uncountable regular cardinal. If $\operatorname{Ext}_{R}^{1}\left(J, M^{(X)}\right)=0$ for every $\leq \kappa$-generated submodule $J \leq Q$ and every set $X$, then $C C(\lambda, M)$ holds for every uncountable regular cardinal $\lambda \leq \kappa$.

In particular, $C C(\lambda, M)$ holds for any uncountable regular $\lambda$ whenever $M$ is $\Sigma$-cotorsion.

Proof. Let $\kappa$ be an uncountable regular cardinal and $M$ be a module such that $\operatorname{Ext}_{R}^{1}\left(J, M^{(X)}\right)=0$ for every $\leq \kappa$-generated submodule $J \leq Q$ and every set $X$. We will prove the statement by induction on $\lambda \leq \kappa$.

Let $\mathfrak{F}=\left(r_{\alpha \beta}\right)_{\alpha<\beta<\lambda}$ be a $\lambda$-factoring system and assume that $C C(\mu, M)$ holds for each regular uncountable $\mu<\lambda$. We want to show that $C C(\mathfrak{F}, M)$ is satisfied. Let us denote $J^{\prime}=\bigcup_{\delta<\lambda} r_{0 \delta}^{-1} R$ and also $J_{\gamma}=\bigcup_{\delta<\gamma} r_{0 \delta}^{-1} R$ for each $\gamma \leq \lambda$. Then clearly $\left(J_{\gamma} \mid \gamma \leq \lambda\right)$ is a $\lambda$-filtration of $J^{\prime}$.

Also by the assumption, $\operatorname{Ext}_{R}^{1}\left(J_{\gamma}, M^{(X)}\right)=0$ for every $\gamma \leq \lambda$ and every set $X$. Hence, we can use Theorem 1.1 for $\mathcal{B}=\left\{M^{(X)} \mid X\right.$ a set $\}$ to obtain a closed unbounded subset $C \subseteq \lambda$ such that:

$$
\operatorname{Ext}_{R}^{1}\left(J_{\beta} / J_{\alpha}, M^{(X)}\right)=0 \quad(\forall \alpha, \beta \in C, \alpha<\beta)(\forall X)
$$

Note that if we intersect $C$ with the set of all limit ordinals less than $\lambda$, we get again a closed unbounded subset of $\lambda$. So we can w.l.o.g. assume that $C$ consists only of limit ordinals. It follows easily from $(*)$ that for every $\gamma \in C$ the map $\operatorname{Hom}_{R}\left(J_{\gamma+1}, M^{(X)}\right) \rightarrow \operatorname{Hom}_{R}\left(J_{\gamma}, M^{(X)}\right)$ is surjective, hence $\operatorname{Ext}_{R}^{1}\left(J_{\gamma+1} / J_{\gamma}, M^{(X)}\right)=0$. Note that by our construction, $J_{\gamma+1}$ is the submodule of $Q$ generated by $r_{0 \gamma}^{-1}$. We will show that the set $C$ fits the definition of $C C(\mathfrak{F}, M)$. Let $\gamma \in C$. Then there are two cases:

First case: $\operatorname{cf} \gamma=\omega$. Choose a sequence $\left(\alpha_{n} \mid n<\omega\right)$ of ordinals (not necessarily from $C$ ) such that $\gamma=\sup _{n<\omega} \alpha_{n}=\sup _{n<\omega}\left(\alpha_{n}+1\right)$. Then by the construction we have:

$$
J_{\gamma+1} / J_{\gamma}=r_{0 \gamma}^{-1} R / \bigcup_{n<\omega} r_{0 \alpha_{n}}^{-1} R=\underline{\lim _{0 \gamma}} r_{0 \gamma}^{-1} R / r_{0 \alpha_{n}}^{-1} R,
$$


where the maps in the direct limit are just the canonical projections. Since $\operatorname{Ext}_{R}^{1}\left(J_{\gamma+1} / J_{\gamma}, M^{(X)}\right)=0$ for any $X$, Lemma 1.2 (1) yields for $X=\omega$ :

$$
\lim ^{1} \operatorname{Hom}_{R}\left(r_{0 \gamma}^{-1} R / r_{0 \alpha_{n}}^{-1} R, M^{(\omega)}\right)=0 .
$$

Hence the inverse system $\left(\operatorname{Hom}_{R}\left(r_{0 \gamma}^{-1} R / r_{0 \alpha_{n}}^{-1} R, M\right)\right)_{n<\omega}$ satisfies the MittagLeffler condition by Theorem 1.3. It is just a matter of easy computation to see that the latter inverse system is isomorphic to the system

$$
M\left[r_{\alpha_{0} \gamma}\right] \supseteq M\left[r_{\alpha_{1} \gamma}\right] \supseteq M\left[r_{\alpha_{2} \gamma}\right] \supseteq \ldots
$$

But this clearly means that the chain $\left(M\left[r_{\alpha_{n} \gamma}\right]\right)_{n<\omega}$ is stationary. Note that since $\left(M\left[r_{\alpha \gamma}\right]\right)_{\alpha<\gamma}$ is a descending chain and we have just proved that it has a cofinal stationary subchain, the whole chain $\left(M\left[r_{\alpha \gamma}\right]\right)_{\alpha<\gamma}$ must be stationary as well.

Second case: $\operatorname{cf} \gamma \geq \aleph_{1}$. Note that this can only happen when $\lambda>\aleph_{1}$. In particular if $\lambda$ is equal to $\aleph_{1}$, then the fact that $C C(\mathfrak{F}, M)$ is satisfied has already been proved by the first case. Otherwise, there are no strictly descending chains of the form $\left(M\left[t_{\alpha}\right]\right)_{\alpha<\aleph_{1}}, t_{\alpha} \in R$, by induction hypothesis for $\lambda=\aleph_{1}$ and by Lemma 3.3.

Assume by way of contradiction that the chain $\left(M\left[r_{\alpha \gamma}\right]\right)_{\alpha<\gamma}$ is not stationary. Then there is a cofinal subset $D$ of $\gamma$ of cardinality of $\gamma$ such that the chain $\left(M\left[r_{\alpha \gamma}\right]\right)_{\alpha \in D}$ is strictly decreasing. But this is impossible as proved before.

Combining previous results together, we get the following corollary:

Corollary 3.5. Let $M$ be an R-module such that $\operatorname{Ext}_{R}^{1}\left(J, M^{(X)}\right)=0$ for every $\leq \aleph_{1}$-generated submodule $J \leq Q$ and every set $X$. Then the set $\{t M \mid t \in R\}$ is countable and well-ordered with respect to inclusion.

Proof. We already know from Proposition 2.1 that $\{t M \mid t \in R\}$ is well ordered. By Lemma 3.4, $C C\left(\aleph_{1}, M\right)$ holds. But then by Lemma 3.3, there is no strictly descending chain of the form $\left(M\left[t_{\alpha}\right]\right)_{\alpha<\aleph_{1}}, t_{\alpha} \in R$. Which in turn implies together with Lemma 2.2 that there is no strictly ascending chain of the form $\left(t_{\alpha} M\right)_{\alpha<\aleph_{1}}, t_{\alpha} \in R$. Hence $\{t M \mid t \in R\}$ is countable.

Now we aim to characterize $\Sigma$-cotorsion modules. More precisely, the goal is to prove that the necessary conditions for $M$ being $\Sigma$-cotorsion given by Proposition 2.1 (well-ordering on $\{t M \mid t \in R\}$ ) and Lemma 3.4 (conditions $C C(\lambda, M))$ are actually sufficient. But first we need a technical lemma.

Lemma 3.6. Let $M$ be a module such that the set $\{t M \mid t \in R\}$ is wellordered, $\kappa$ be an uncountable regular cardinal, and $\mathfrak{F}=\left(r_{\alpha \beta}\right)_{\alpha<\beta<\kappa}$ be a $\kappa$-factoring system. Then there is a closed unbounded subset $C \subseteq \kappa$ with the following property: For each $\beta \in C$ and for each $\alpha<\beta$, there exists an ordinal number $\xi$ such that $\alpha<\xi<\beta$ and $r_{\alpha \gamma} M=r_{\alpha \xi} M$ for each $\gamma \geq \xi$.

Proof. Define a function $s: \kappa \rightarrow \kappa$ by

$$
s(\alpha)=\min \left\{\delta>\alpha \mid r_{\alpha \delta} M=r_{\alpha \gamma} M \text { for each } \gamma \geq \delta\right\}
$$

Note that $s$ is well-defined since $\{t M \mid t \in R\}$ satisfies d.c.c. with respect to inclusion. We claim that $s$ is an increasing function. Let $\alpha<\beta<\kappa$ and choose arbitrarily $\gamma \geq s(\beta)$. Then $r_{\beta s(\beta)} M=r_{\beta \gamma} M$ by definition. After 
multiplying both sides of this equality by $r_{\alpha \beta}$, we get $r_{\alpha s(\beta)} M=r_{\alpha \gamma} M$. Hence $s(\alpha) \leq s(\beta)$, again by definition.

Next, we will inductively construct a strictly increasing continuous sequence of ordinals $\left(d_{\zeta}\right)_{\zeta<\kappa}$ such that $d_{\zeta}<\kappa$ for each $\zeta<\kappa$. Put

$$
d_{0}=\sup \{0, s(0), s(s(0)), \ldots\}=\sup _{n<\omega} s^{n}(0)
$$

For a limit ordinal $\zeta$ we put $d_{\zeta}=\sup _{\eta<\zeta} d_{\eta}$, and for ordinal successors we define

$$
d_{\eta+1}=\sup \left\{d_{\eta}, s\left(d_{\eta}\right), s\left(s\left(d_{\eta}\right)\right), \ldots\right\}=\sup _{n<\omega} s^{n}\left(d_{\eta}\right)
$$

It is easy to see that the set $C=\left\{d_{\zeta} \mid \zeta<\kappa\right\}$ is closed unbounded in $\kappa$. Let $\beta \in C$; that is, $\beta=d_{\zeta}$ for some $\zeta<\kappa$. We will show that for any $\alpha<\beta$ also $s(\alpha)<\beta$ and thus $r_{\alpha \gamma} M=r_{\alpha s(\alpha)} M$ whenever $s(\alpha) \leq \gamma<\kappa$. Putting $\xi=s(\alpha)$ will then conclude the proof. We will consider two cases:

First case: $\zeta=0$ or $\zeta=\eta+1$ for some $\eta<\kappa$. Let $c=0$ if $\zeta=0$ and $c=d_{\eta}$ if $\zeta=\eta+1$. By definition of $d_{\zeta}$, there is a natural number $n$ such that $\alpha<s^{n}(c)$. Hence $s(\alpha) \leq s^{n+1}(c)<d_{\zeta}=\beta$.

Second case: $\zeta$ is a limit ordinal. Then there is $\eta<\zeta$ such that $\alpha<d_{\eta+1}$. And by the first case $s(\alpha)<d_{\eta+1}<d_{\zeta}=\beta$.

Now, we are ready to prove a general result from which we will easily derive the characterization of $\Sigma$-cotorsion modules.

Theorem 3.7. Let $R$ be a valuation domain, $M$ an $R$-module and $\kappa$ an infinite regular cardinal. Then the following are equivalent:

(1) $\operatorname{Ext}_{R}^{1}\left(J, M^{(X)}\right)=0$ for every $\leq \kappa$-generated $R$-submodule $J \leq Q$ and every set $X$.

(2) The set $\{t M \mid t \in R\}$ is well-ordered with respect to inclusion and the condition $C C(\lambda, M)$ is satisfied for every regular uncountable cardinal $\lambda \leq \kappa$.

Proof. The implication (1) $\Rightarrow$ (2) follows by Proposition 2.1 and Lemma 3.4.

Conversely, assume that (2) holds for some $\kappa$. Let $J \leq Q$ be a $\leq \kappa$ generated $R$-submodule of $Q$. We have to prove that $\operatorname{Ext}_{R}^{1}\left(J, M^{(X)}\right)=0$ for every set $X$. If $J$ is countably generated, this follows by Proposition 2.1. We will proceed further by induction on the minimal number of generators of $J$.

Assume that gen $J=\lambda \leq \kappa$ for some $\lambda$ uncountable and $\operatorname{Ext}_{R}^{1}\left(J^{\prime}, M^{(X)}\right)=$ 0 for every $<\lambda$-generated submodule $J^{\prime} \leq Q$ and every set $X$. Then, as mentioned just after Definition 3.1, there is a $\kappa$-factoring system $\mathfrak{F}=\left(r_{\alpha \beta}\right)_{\alpha<\beta<\lambda}$ such that $J \cong \bigcup_{\eta<\lambda} r_{0 \eta}^{-1} R$. Let us also denote $J_{\zeta}=\bigcup_{\eta<\zeta} r_{0 \eta}^{-1} R$ for each $\zeta \leq \lambda$. Then clearly $\left(J_{\zeta} \mid \zeta \leq \lambda\right)$ is a $\lambda$-filtration of $J_{\lambda} \cong J$ and $J_{\zeta+1}=r_{0 \zeta}^{-1} R$ for each $\zeta<\lambda$.

Let $C \subseteq \lambda$ be the closed unbounded subset given by the condition $C C(\mathfrak{F}, M)$. Since we can intersect $C$ with the closed unbounded set given by Lemma 3.6 and take the intersection instead of the original $C$, we can without loss of generality assume that

for each $\gamma \in C$ and $\alpha<\gamma$, there exists $\xi=\xi(\alpha)$ such that

$$
\alpha<\xi<\gamma \text { and } r_{\alpha \delta} M=r_{\alpha \xi} M \text { for each } \delta \geq \xi \text {. }
$$


For a similar reason, we can assume that $C$ only contains limit ordinals.

Now fix $\gamma, \delta \in C, \gamma<\delta$, and look at the direct system $\left(r_{0 \delta}^{-1} R / r_{0 \alpha}^{-1} R\right)_{\alpha<\gamma}=$ $\left(J_{\delta+1} / J_{\alpha+1}\right)_{\alpha<\gamma}$ where the maps are the canonical projections. If we apply the functor $\operatorname{Hom}_{R}\left(-, M^{(X)}\right)$ to it, we get the corresponding inverse system $\left(\operatorname{Hom}_{R}\left(r_{0 \delta}^{-1} R / r_{0 \alpha}^{-1} R, M^{(X)}\right)\right)_{\alpha<\gamma}$ which is easily shown to be isomorphic to the inverse system $\left(M\left[r_{\alpha \delta}\right]^{(X)}\right)_{\alpha<\gamma}$ with just the inclusions as the maps. But since the descending chain $\left(M\left[r_{\alpha \gamma}\right]\right)_{\alpha<\gamma}$ is stationary by assumption and $M\left[r_{\alpha \delta}\right]=r_{\gamma \delta}^{-1} M\left[r_{\alpha \gamma}\right]$, the chain $\left(M\left[r_{\alpha \delta}\right]^{(X)}\right)_{\alpha<\gamma}$ must be stationary as well for any $X$. In other words, the latter chain has a constant cofinal subchain, and since $\lim ^{1}$ does not change when we pass to a cofinal subchain (cf. [20, Theorem $\overleftarrow{\mathrm{B}])}$, Theorem 1.5 yields:

$$
\varliminf_{\alpha<\gamma} \lim ^{1} \operatorname{Hom}_{R}\left(r_{0 \delta}^{-1} R / r_{0 \alpha}^{-1} R, M^{(X)}\right) \cong \lim _{\alpha<\gamma}^{1} M\left[r_{\alpha \delta}\right]^{(X)}=0 .
$$

Next look at the direct system of short exact sequences $0 \rightarrow r_{0 \alpha}^{-1} R \rightarrow$ $r_{0 \delta}^{-1} R \rightarrow r_{0 \delta}^{-1} R / r_{0 \alpha}^{-1} R \rightarrow 0$ indexed by ordinals $\alpha<\gamma$ with the obvious maps. If we apply the functor $\operatorname{Hom}_{R}\left(-, M^{(X)}\right)$ to this system, we get an inverse system of exact sequences

$$
\begin{aligned}
0 \rightarrow \operatorname{Hom}_{R}\left(r_{0 \delta}^{-1} R / r_{0 \alpha}^{-1} R, M^{(X)}\right) & \rightarrow \\
& \rightarrow \operatorname{Hom}_{R}\left(r_{0 \delta}^{-1} R, M^{(X)}\right) \rightarrow \operatorname{Hom}_{R}\left(r_{0 \alpha}^{-1} R, M^{(X)}\right)
\end{aligned}
$$

which is easily seen to be isomorphic to the inverse system of exact sequences

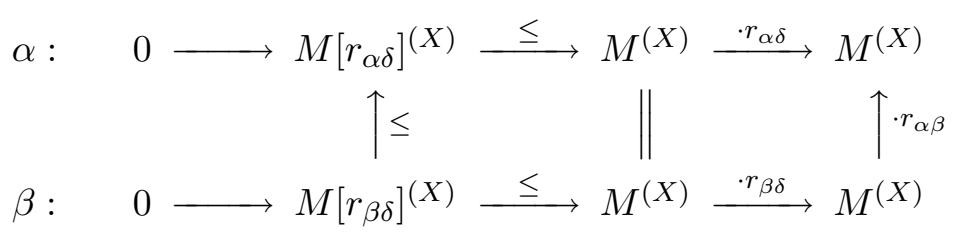

where $\alpha<\beta<\gamma$. We claim that the following sequence which arises just by taking inverse limit of the latter inverse system is exact:

$$
0 \rightarrow \bigcap_{\alpha<\gamma} M\left[r_{\alpha \delta}\right]^{(X)} \rightarrow M^{(X)} \rightarrow \lim _{\alpha<\gamma} M^{(X)} \rightarrow 0 .
$$

To see this, note first that by $(\ddagger)$, the inverse limit of the inverse system of the short exact sequences $0 \rightarrow M\left[r_{\alpha \delta}\right]^{(X)} \rightarrow M^{(X)} \stackrel{p_{\alpha}}{\longrightarrow} r_{\alpha \delta} M^{(X)} \rightarrow 0$ is exact. Therefore, since the maps $M^{(X)} \stackrel{\cdot r_{\alpha \delta}}{\longrightarrow} M^{(X)}$ factor as $M^{(X)} \stackrel{p_{\alpha}}{\longrightarrow}$ $r_{\alpha \delta} M^{(X)} \leqq M^{(X)}$, we only need to prove that the limit of the inverse system of the inclusions

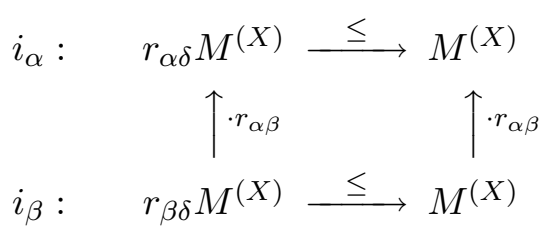

is an isomorphism. By left exactness of inverse limits, the limit map $\varliminf_{\alpha<\gamma} i_{\alpha}$ must be injective. Conversely, let $m$ be an element in the codomain of $\lim _{\alpha<\gamma} i_{\alpha}$; that is, $m$ can be viewed as a sequence $\left(m_{\alpha}\right)_{\alpha<\gamma} \in\left(M^{(X)}\right)^{\gamma}$ such that $m_{\alpha}=r_{\alpha \beta} m_{\beta}$ for each $\alpha<\beta<\gamma$. But then for each $\alpha<\gamma$, there exists $\xi$ such that $\alpha<\xi<\gamma$ and $r_{\alpha \delta} M^{(X)}=r_{\alpha \xi} M^{(X)}$. This follows from $(a)$ and 
the assumption that $\gamma \in C$. In particular, $m_{\alpha}=r_{\alpha \xi} m_{\xi} \in r_{\alpha \delta} M^{(X)}$ for each $\alpha<\gamma$ and thus $\left(m_{\alpha}\right)_{\alpha<\gamma}$ is actually an element of $\lim _{\alpha<\gamma} r_{\alpha \delta} M^{(X)}$. Hence $\lim _{\alpha<\gamma} i_{\alpha}$ is surjective as well and the claim is proved.

By applying the conclusion of the claim to the isomorphic inverse system of exact sequences $(b)$, we get an exact sequence

$$
\begin{aligned}
& 0 \rightarrow \lim _{\alpha<\gamma} \operatorname{Hom}_{R}\left(r_{0 \delta}^{-1} R / r_{0 \alpha}^{-1} R, M^{(X)}\right) \rightarrow \\
& \quad \rightarrow \underbrace{\lim }_{\alpha<\gamma} \operatorname{Hom}_{R}\left(r_{0 \delta}^{-1} R, M^{(X)}\right) \rightarrow \lim _{\alpha<\gamma} \operatorname{Hom}_{R}\left(r_{0 \alpha}^{-1} R, M^{(X)}\right) \rightarrow 0
\end{aligned}
$$

By using the well-known fact that contravariant Hom-functors take direct limits to inverse limits and the definition of the modules $J_{\zeta}, \zeta<\lambda$, we see that the last exact sequence is isomorphic to

$0 \rightarrow \operatorname{Hom}_{R}\left(J_{\delta+1} / J_{\gamma}, M^{(X)}\right) \rightarrow \operatorname{Hom}_{R}\left(J_{\delta+1}, M^{(X)}\right) \rightarrow \operatorname{Hom}_{R}\left(J_{\gamma}, M^{(X)}\right) \rightarrow 0$

We conclude that the map $\operatorname{Hom}_{R}\left(J_{\delta+1}, M^{(X)}\right) \rightarrow \operatorname{Hom}_{R}\left(J_{\gamma}, M^{(X)}\right)$ coming from the inclusion $J_{\gamma} \leq J_{\delta+1}$ is surjective. Hence also the map

$$
\operatorname{Hom}_{R}\left(J_{\delta}, M^{(X)}\right) \rightarrow \operatorname{Hom}_{R}\left(J_{\gamma}, M^{(X)}\right)
$$

is surjective, and since $J_{\delta}$ is $<\lambda$-generated and we have $\operatorname{Ext}_{R}^{1}\left(J_{\delta}, M^{(X)}\right)=0$ by inductive hypothesis, it follows $\operatorname{Ext}_{R}^{1}\left(J_{\delta} / J_{\gamma}, M^{(X)}\right)=0$.

Finally, the chain $\left(J_{\zeta} \mid \zeta \in C \cup\{\lambda\}\right)$ is a filtration of $J_{\lambda} \cong J$ with the property that $\operatorname{Ext}_{R}^{1}\left(J_{\beta} / J_{\alpha}, M^{(X)}\right)=0$ for each $\alpha, \beta \in C, \alpha<\beta$ and for each set $X$. It is a well-known result that $\operatorname{Ext}_{R}^{1}\left(J, M^{(X)}\right)=0$ for each set $X$ in this case (see for example [9, Proposition XII.1.14]).

Now we formulate the promised characterization of $\Sigma$-cotorsion modules.

Theorem 3.8. Let $R$ be a valuation domain. An $R$-module $M$ is $\Sigma$-cotorsion if and only if

(1) The family $\{t M \mid t \in R\}$ of submodules of $M$ is (countable and) well-ordered with respect to inclusion.

(2) The condition $C C(\kappa, M)$ is satisfied for every uncountable regular cardinal $\kappa$.

Proof. This is an immediate consequence of Theorem 3.7 taking into account the fact that a module $N$ is cotorsion if and only if $\operatorname{Ext}_{R}^{1}(J, N)=0$ for every submodule $J \leq Q$. If either of the equivalent conditions holds, then the set $\{t M \mid t \in R\}$ is necessarily countable by Corollary 3.5.

As an important application of Theorem 3.8, we prove one of the main results of this paper, which includes an analogue of [13, Corollaries 13 and 14] and answers the question posed in [13] on whether a pure submodule of a $\Sigma$-cotorsion module is always cotorsion, both in the setting of modules over a valuation domain.

Theorem 3.9. Let $R$ be a valuation domain and $M$ be a $\Sigma$-cotorsion $R$ module. Then the following modules are again $\Sigma$-cotorsion over $R$ :

(a) any pure submodule of $M$,

(b) any pure-epimorphic image of $M$, 
(c) any direct product of copies of $M$,

(d) any module elementarily equivalent to $M$,

(e) any module of the form $r M, r \in R$,

(f) any module of the form $M / r M, r \in R$.

Proof. Clearly, $N[s]=M[s] \cap N$ for every submodule $N$ of $M$. Hence if $M$ is $\Sigma$-cotorsion, every submodule $N$ satisfies condition (2) of Theorem 3.8. If $N$ is a pure submodule of $M$, then $s N=s M \cap N$ for every $s \in R$ and condition (1) of Theorem 3.8 is inherited by $N$. Hence $N$ is $\Sigma$-cotorsion and (a) follows. The same conclusion applies to every submodule of the form $r M$ for $r \in R$, since the set $\{t(r M) \mid t \in R\}$ is a subset of $\{t M \mid t \in R\}$. Hence (e) follows. Next, (b) and (f) follow from (a) and (e), respectively, by the fact that cotorsion modules are closed under taking cokernels of monomorphisms. For (c), the conclusion follows again by Theorem 3.8, noting that $r\left(M^{X}\right)=(r M)^{X}$ and $\left(M^{X}\right)[r]=(M[r])^{X}$ for every $r \in R$ and every set $X$. Finally, if $N$ is elementarily equivalent to $M$, then $N$ is a pure submodule of an ultraproduct of copies of $M$ by the theorem of Frayne [8, Corollary 4.3.13]. Hence $N$ is $\Sigma$-cotorsion by (c), (b) and (a), and (d) follows.

\section{Divisible $\Sigma$-COTORSiOn MOdules}

In this section, we characterize divisible $\Sigma$-cotorsion modules over valuation domains $R$. There are two main reasons for this. First, it is much easier to deal with divisible modules than with all modules. In particular the set $\{t D \mid t \in R\}$ is equal to $\{0, D\}$ for $D$ divisible, hence it is obviously wellordered. The other reason is deeper - it turns out that divisible $\Sigma$-cotorsion modules are building blocks of all $\Sigma$-cotorsion modules as we will see in the next section. We start with an easy observation.

Lemma 4.1. Let $D$ be a divisible $R$-module and $P=D_{\#}$. Then the sets $\left\{t\left(R_{P}\right) \mid t \in R\right\}$ and $\{D[t] \mid t \in R\}$ are antiisomorphic totally ordered sets (both with respect to inclusion), and the corresponding order antiisomorphism is given by sending $t\left(R_{P}\right)$ to $D[t]$.

Proof. Since $R$ is a valuation domain, the localization $R_{P}$ is again a valuation domain and both $\left\{t\left(R_{P}\right) \mid t \in R\right\}$ and $\{D[t] \mid t \in R\}$ are totally ordered with respect to inclusion. We need to prove that $t\left(R_{P}\right)<s\left(R_{P}\right)$ if and only if $D[t]>D[s]$. Note that $D^{\#}=0$, hence $D$ is in fact an $R_{P}$-module. But then $t\left(R_{P}\right) \geq s\left(R_{P}\right)$ implies $D[t] \leq D[s]$, so the if part is done. For the only if part, note that $t\left(R_{P}\right)<s\left(R_{P}\right)$ implies that $t=s r$ for some $r \in P$. Thus $r \in(s D)_{\#}=D_{\#}$ since $s$ is clearly nonzero, and $D[t]=D[s r]>D[s]$ follows by Lemma 1.10 .

We need one more lemma, which will also be useful later. We prove it, therefore, in a slightly more general setting than just for divisible modules.

Lemma 4.2. Let $M$ be a nonzero $R$-module such that $\operatorname{Ext}_{R}^{1}\left(J, M^{(X)}\right)=0$ for every $\leq \aleph_{1}$-generated $R$-submodule $J \leq Q$ and every set $X$, and denote $P=M_{\#}$ and $L=M^{\#}$. Then all $R_{P}$-submodules $K \leq Q$ such that $L<$ $K \leq R_{L}$ are countably generated (as $R_{P}$-modules). 
Proof. Note first that the assumption on $M$ is equivalent to $\{t M \mid t \in$ $R\}$ being well-ordered and the condition $C C\left(\aleph_{1}, M\right)$ being satisfied (see Theorem 3.7). Hence $L \leq P$ by Lemma 2.2.

Assume now by way of contradiction that there is an uncountably generated $R_{P}$-submodule $K \leq Q$ such that $L<K \leq R_{L}$. That is, $K=$ $\bigcup_{\alpha<\kappa} t_{\alpha} R_{P}$ for some uncountable regular cardinal $\kappa$ and a strictly ascending chain $\left(t_{\alpha} R_{P}\right)_{\alpha<\kappa}$. We can w.l.o.g. assume that $t_{\alpha} \in R_{L} \backslash L$ for all $\alpha<\kappa$ and, by passing from $K$ to $\bigcup_{\alpha<\aleph_{1}} t_{\alpha} R_{P}$, that $\kappa=\aleph_{1}$. If we put $r_{\alpha \beta}=t_{\alpha} t_{\beta}^{-1}$, we get an $\aleph_{1}$-factoring system $\mathfrak{F}=\left(r_{\alpha \beta}\right)_{\alpha<\beta<\aleph_{1}}$ such that $r_{\alpha \beta} \in P \backslash L$ for each $\alpha<\beta<\aleph_{1}$. But then $M\left[r_{\alpha \gamma}\right]>M\left[r_{\beta \gamma}\right]$ for each $\alpha<\beta<\gamma<\kappa$ by Lemma 1.10 since $r_{\alpha \beta} \in P=M_{\#}=\left(r_{\beta \gamma} M\right)_{\#}$. In other words, condition $C C(\mathfrak{F}, M)$ is not satified, which contradicts the assumptions.

Now we are in a position to give the characterization.

Theorem 4.3. Let $R$ be a valuation domain, $D$ be a nonzero divisible $R$ module and $P=D_{\#}$. Then the following are equivalent:

(1) $D$ is $\Sigma$-cotorsion.

(2) $\operatorname{Ext}_{R}^{1}\left(J, D^{(X)}\right)=0$ for every $\leq \aleph_{1}$-generated $R$-submodule $J \leq Q$ and every set $X$.

(3) Condition $C C\left(\aleph_{1}, D\right)$ is satisfied.

(4) There is neither a strictly descending nor a strictly ascending chain of the form $\left(D\left[t_{\alpha}\right]\right)_{\alpha<\aleph_{1}}, t_{\alpha} \in R$.

(5) $Q$ has no uncountably generated $R_{P}$-submodules.

Proof. (1) $\Longrightarrow(2)$ is clear.

$(2) \Longrightarrow(5)$. This follows from Lemma 4.2 since $D^{\#}=0$ for $D$ nonzero divisible.

$(5) \Longrightarrow(1)$. This is an immediate consequence of Lemma 2.5 (2), Proposition 2.1 and the fact that $\left\{t D \mid t \in R_{P}\right\}=\{0, D\}$ is obviously well-ordered.

$(2) \Longleftrightarrow(3)$. This follows directly from Theorem 3.7 used for $\kappa=\aleph_{1}$, again using the fact that $\{t D \mid t \in R\}=\{0, D\}$.

(4) $\Longleftrightarrow(5)$. Assume that there is a chain $\left(D\left[t_{\alpha}\right]\right)_{\alpha<\aleph_{1}}$ that is either strictly ascending or strictly descending. Then the chain $\left(t_{\alpha} R_{P}\right)_{\alpha<\aleph_{1}}$ is either strictly descending or strictly ascending by Lemma 4.1. Hence, either $\bigcup_{\alpha<\aleph_{1}} t_{\alpha} R_{P}$ or $\bigcup_{\alpha<\aleph_{1}} t_{\alpha}^{-1} R_{P}$ is an uncountably generated $R_{P \text {-submodule of }}$ $Q$.

On the other hand, if $J \leq Q$ is an uncountably generated $R_{P}$-submodule, then $J$ is a union of a strictly ascending chain of the form $\bigcup_{\alpha<\kappa} t_{\alpha} R_{P}$ or $\bigcup_{\alpha<\kappa} t_{\alpha}^{-1} R_{P}$ if $J<R$ or $J>R$, respectively. Hence $\left(t_{\alpha} R_{P}\right)_{\alpha<\aleph_{1}}$ is either strictly ascending or strictly descending, and so is $\left(D\left[t_{\alpha}\right]\right)_{\alpha<\aleph_{1}}$ by Lemma 4.1.

Remark. Note that there is no restriction on the prime ideals which we can get as $D_{\#}$ for $D$ divisible. More precisely, if $P$ is any prime ideal, then $D=Q / R_{P}$ is a divisible $R$-module with $D_{\#}=P$. We can even find an injective module $D$ with $D_{\#}=P$, namely $D=\operatorname{Hom}_{\mathbb{Z}}\left(R_{P}, \mathbb{Q} / \mathbb{Z}\right)$.

The previous theorem also gives us an easy way to construct valuation domains with divisible $\Sigma$-cotorsion modules that are not $\Sigma$-pure-injective, 
and on the other hand domains with divisible modules that are not $\Sigma$ cotorsion.

Example 4.4. Let $\kappa$ be a cardinal and consider a valuation domain $R$ such that the value group is isomorphic to $\mathbb{Z}^{(\kappa)}$ with lexicographic ordering. Put $D=Q / R$.

If $\kappa=\aleph_{0}$, then $D$ is clearly $\Sigma$-cotorsion by Theorem 4.3 , since $\mathbb{Z}^{\left(\aleph_{0}\right)}$ is a countable set. But the maximal ideal of $R$ is $\aleph_{0}$-generated, hence by Lemma 4.1 there is a strictly descending chain of the form

$$
D\left[t_{0}\right] \supsetneqq D\left[t_{1}\right] \supsetneqq D\left[t_{2}\right] \supsetneqq \cdots
$$

All the groups $D\left[t_{i}\right]$ are finitely definable subgroups of $D$, hence, by the well known characterization of $\Sigma$-pure-injective modules proved in [14] or [27], the module $D$ cannot be $\Sigma$-pure-injective.

If $\kappa=\aleph_{1}$, then the maximal ideal $J$ of $R$ is $\aleph_{1}$-generated, so Lemma 4.1 yields a strictly descending chain of the form $\left(D\left[t_{\alpha}\right]\right)_{\alpha<\aleph_{1}}$. Hence $D$ is not $\Sigma$-cotorsion by Theorem 4.3. In fact, one can check that $\operatorname{Ext}_{R}^{1}\left(J, D^{\left(\aleph_{1}\right)}\right) \neq 0$.

The above examples give us a strong indication that the concept of $\Sigma$ cotorsion modules is much harder to describe than the concept of $\Sigma$-pure injective modules. Namely, the property of being $\Sigma$-cotorsion is in general not of countable character in the sense of Guil-Asensio and Herzog [13]:

Proposition 4.5. Let $R$ be a valuation domain with the value group isomorphic to $\mathbb{Z}^{\left(\aleph_{1}\right)}$ with lexicographic ordering. Then the module $D=Q / R$ is not $\Sigma$-cotorsion as an $R$-module, but its restriction ${ }_{S} D$ to any countable subring $S$ of $R$ is a $\Sigma$-cotorsion $S$-module.

Proof. By the above example $D$ is not a $\Sigma$-cotorsion $R$-module. On the other hand, if $S$ is a countable subring of $R$, then $S$ is certainly a domain and $D$ has an induced structure of a divisible $S$-module. By Proposition 1.9 the restriction ${ }_{S} D$ of $D$ to $S$ is a $\Sigma$-cotorsion $S$-module.

\section{Structure of $\Sigma$-COTORsion Modules}

In this last section, we will investigate the structure of general $\Sigma$-cotorsion modules over valuation domains. We show that this depends very much on the structure of divisible $\Sigma$-cotorsion modules characterized in the previous section. The first observation to start with is that it is enough to investigate the structure of torsion $\Sigma$-cotorsion modules.

Lemma 5.1. Let $M$ be a $\Sigma$-cotorsion module. Then $M \cong T \oplus Q^{(\gamma)}$ where $T$ is a torsion $\Sigma$-cotorsion module.

Proof. By [12, XIII 8.8], any cotorsion module $M$ is decomposed into a direct sum $M_{1} \oplus F$ where $M_{1}$ is the cotorsion envelope of its torsion submodule and $F$ is a torsion-free cotorsion module, hence pure-injective (by $[12$, I.8.11, XIII.8.]). Thus, if $M$ is $\Sigma$-cotorsion, then $F$ is $\Sigma$-pure injective. But $F_{\#}=0$, so $F$ is a $Q$-module by Lemma 2.5 (2) used for $P=0$. Hence $F \cong Q^{(\gamma)}$ for some set $\gamma$. The summand $M_{1}$ is also $\Sigma$-cotorsion and by Theorem 3.9 we conclude that the torsion submodule of $M_{1}$ is cotorsion since a module is always a pure submodule of its cotorsion envelope. Hence $M_{1}$ coincides with its torsion submodule. 
Next, we will follow a slightly different line of investigation, which is somewhat similar to the case of Dedekind domains. But unlike for Dedekind domains, we get a non-split exact sequence in general, as explained after the proof of the proposition.

Proposition 5.2. Let $M$ be an $R$-module. $M$ is $\Sigma$-cotorsion $R$-module if and only if there is a short exact sequence

$$
0 \rightarrow D \rightarrow M \rightarrow B \rightarrow 0
$$

where $D$ is a divisible $\Sigma$-cotorsion module of the form $t M$ for some element $t \in R$ and $B$ is bounded $\Sigma$-cotorsion.

Proof. Sufficiency is clear. Let $M$ be $\Sigma$-cotosion. Then the set $\mathcal{S}=\{t M \mid$ $0 \neq t \in R\}$ has the smallest element by Proposition 2.1. Denote this smallest element by $D$ and put $B=M / D$. Then $D=t M$ for some $t$ and $s D=$ $s t M=t M=D$ by minimality for each $0 \neq s \in R$. Hence $D$ is divisible (whether or not it is a zero module) and $B$ is bounded. Both $D$ and $B$ are $\Sigma$-cotorsion by Theorem 3.9.

Remark. The exact sequence in Proposition 5.2 need not split. This can be shown by considering the class $I C$ of valuation domains introduced in [5] ( $I C$ stands for incompleteness condition). They have the property that all submodules of $Q$ are countably generated and also that $\operatorname{Ext}_{R}^{1}(R / I, K) \neq 0$ for $K=Q / R$ and every non-principal ideal $I$ of $R$. Examples of such domains are given in [5, Ex. 2.5 and 2.6]. If $R$ is an $I C$-domain with a nonprincipal maximal ideal $P$ (see [5, Ex. 2.6]), then there is a non-splitting exact sequence $0 \rightarrow K \rightarrow T \rightarrow R / P \rightarrow 0$, where $K$ is divisible torsion and $R / P$ is bounded. By Proposition 2.1 , both $K$ and $R / P$ are $\Sigma$-cotorsion, so $T$ is $\Sigma$-cotorsion.

In order to obtain the structure of bounded $\Sigma$-cotorsion modules, we state a preliminary lemma.

Lemma 5.3. Let $M$ be an $R$-module and $I$ an ideal of $R$. The following hold:

(1) If $0<I M$, then $(I M)^{\#} \leq M^{\#}$.

(2) If $I M<M$, then $(M / I M)^{\#}=M^{\#}$.

(3) If the set $\{t M \mid t \in R\}$ is well-ordered with respect to inclusion, then the set $\{t I M \mid t \in R\}$ is well-ordered with respect to inclusion.

(4) If $M$ is $\Sigma$-cotorsion, then both $I M$ and $M / I M$ are $\Sigma$-cotorsion.

Proof. (1) Let $r \notin M^{\#}$, that is $r M=M$. Then also $r I M=I M$, hence $r \notin(I M)^{\#}$.

(2) Let $r \in(M / I M)^{\#}$, then $r M+I M<M$; hence also $r M<M$, that is $r \in M^{\#}$. Conversely, let $r \in M^{\#}$. If $r R \leq I$ then $r M+I M=I M$, hence $r(M / I M)=0$. If $I<r R$ then $r M+I M=r M$, hence $r(M / I M)<M / I M$. In both cases $r \in(M / I M)^{\#}$.

(3) We may assume that $I M \neq 0$. Assume by way of contradiction that there exists a strictly descending chain

$$
t_{0} I M>t_{1} I M>t_{2} I M>\ldots
$$


Then $t_{0} R>t_{1} R>t_{2} R>\ldots$ is strictly descending, too. Since the set $\{t M \mid$ $t \in R\}$ is well-ordered by assumption, there exists an index $m$ such that $t_{m} M=t_{k} M$ for every $k \geq m$. But this clearly implies that $t_{m} I M=t_{k} I M$ for every $k \geq m$, a contradiction.

(4) It is enough to show that $I M$ satisfies conditions (1) and (2) of Theorem 3.8. The above paragraph shows that condition (1) holds for $I M$. Condition (2) is inherited by all submodules $N$ of $M$, since $M[r] \cap N=N[r]$ for all $r \in R$. Hence, $I M$ and consequently also $M / I M$ are $\Sigma$-cotorsion.

Now, we can state the main theorem concerning the structure of $\Sigma$ cotorsion modules. Using it, we are able to filter any $\Sigma$-cotorsion module by modules which are divisible $\Sigma$-cotorsion over some (valuation) factor domains of $R$. Recall that such divisible $\Sigma$-cotorsion modules are described in Theorem 4.3. Note that it is implicit in the theorem below, in view of Lemma 2.5, that all the composition factors $M_{\alpha+1} / M_{\alpha}$ are $\Sigma$-cotorsion as $R$-modules, too.

Theorem 5.4. Let $R$ be a valuation domain and $M$ be a nonzero $\Sigma$-cotorsion $R$-module. Then there is a countable ordinal $\tau$, a filtration $\left(M_{\alpha} \mid \alpha \leq \tau\right)$ of $M$ and an ascending chain $\left(L_{\alpha}\right)_{\alpha<\tau}$ of prime ideals of $R$ such that for every $\alpha<\tau$ :

(1) $M_{\alpha}$ is a $\Sigma$-cotorsion $R$-module.

(2) $M_{\alpha+1} / M_{\alpha}$ is a nonzero divisible $\Sigma$-cotorsion $R / L_{\alpha}$-module.

Moreover, for any non-limit ordinal $\alpha \leq \tau$ there is $t \in R$ such that $M_{\alpha}=$ $t M$, and conversely for each $t \in R$ there is a unique (possibly limit) ordinal $\alpha \leq \tau$ such that $t M=M_{\alpha}$.

Proof. We will define the filtration $\left(M_{\alpha} \mid \alpha \leq \tau\right)$ by induction on $\alpha$ and in such a way that $M_{\alpha}=I_{\alpha} M$ for some ideal $I_{\alpha} \leq R$. Moreover, $I_{\alpha}$ will be a principal ideal for $\alpha$ non-limit.

We start with $M_{0}=0$ and $I_{0}=0$. For a limit ordinal $\alpha$, we just take $M_{\alpha}=\bigcup_{\beta<\alpha} M_{\beta}$ and $I_{\alpha}=\bigcup_{\beta<\alpha} I_{\beta}$. We stress at this place that $\alpha$ cannot be equal to $\aleph_{1}$ since otherwise $M$ would admit a strictly ascending chain of submodules of the form $\left(s_{\beta+1} M\right)_{\beta<\aleph_{1}}$ where $I_{\beta+1}=s_{\beta+1} R$, a contradiction to Corollary 3.5. If $\alpha=\beta+1$ is an ordinal successor and $M_{\beta}<M$ has been constructed, let $s_{\alpha} M$ be the minimal element of the set $\{t M \mid t \in R \& t M>$ $\left.M_{\beta}\right\}$; this must exist by Proposition 2.1. We put $M_{\alpha}=s_{\alpha} M$ and $I_{\alpha}=s_{\alpha} R$. Clearly, this construction must stop, say for $M_{\tau}=M$, and by the argument above it cannot happen that $\aleph_{1} \leq \tau$. Hence $\tau$ is countable.

Note that the filtration $\left(M_{\alpha} \mid \alpha \leq \tau\right)$ we have just constructed is nothing else than the closure of the well-ordered countable set $\{t M \mid t \in R\}$ under unions of chains.

We define $L_{\alpha}=\left(M_{\alpha+1} / M_{\alpha}\right)^{\#}$. Using Lemmas 5.3 and 2.3, we obtain for each $\alpha \leq \gamma<\tau$ the inequality:

$$
L_{\alpha}=M_{\alpha+1}^{\#}=\left(s_{\alpha+1} M\right)^{\#} \leq\left(s_{\gamma+1} M\right)^{\#}=M_{\gamma+1}^{\#}=L_{\gamma},
$$

showing that $\left(L_{\alpha}\right)_{\alpha<\tau}$ is an ascending chain of prime ideals.

Now, (1) follows from Lemma 5.3, since $M_{\alpha}=I_{\alpha} M$ for some ideal $I_{\alpha}$ by the construction. It follows that $M_{\alpha+1} / M_{\alpha}$ is a $\Sigma$-cotorsion $R$-module 
for each $\alpha<\tau$, since the class of cotorsion modules is closed under cokernels of monomorphisms. Moreover, $t M_{\alpha+1}=t s_{\alpha+1} M$ is either equal to $M_{\alpha+1}$ or contained in $M_{\alpha}$ by construction, hence $L_{\alpha}=\left(M_{\alpha+1} / M_{\alpha}\right)^{\#}=$ $\operatorname{Ann}_{R}\left(M_{\alpha+1} / M_{\alpha}\right)$ and $M_{\alpha+1} / M_{\alpha}$ is a divisible $R / L_{\alpha}$-module. Then (2) follows by Lemma 2.5 .

From the last two statements, the first follows directly by the construction. For the second, let $t \in R$ and consider the set $\mathcal{T}=\left\{\beta \leq \tau \mid t M \leq M_{\beta}\right\}$. $\mathcal{T}$ is non empty, thus $\mathcal{T}$ has a minimal element $\alpha$. It is not difficult to see that $t M=M_{\alpha}$.

We devote the rest of the section to answering the question whether one can state a converse of Theorem 5.4. That is, whether the fact that $M$ has a certain filtration by $\Sigma$-cotorsion modules which are divisible over suitable factor domains implies that $M$ itself is $\Sigma$-cotorsion. One obvious case is when the filtration is finite. Another case is when $M$ is bounded and $R$ is $\aleph_{0}$-noetherian, that is, all ideals of $R$ are countably generated. We know in this case that a bounded module $M$ over an $\aleph_{0}$-noetherian valuation domain is $\Sigma$-cotorsion if and only if $\{t M \mid t \in R\}$ is well-ordered. This follows by Proposition 2.1 and the fact that $\operatorname{Ext}_{R}^{1}\left(Q, M^{(X)}\right)=0$, since $M^{(X)}$ is bounded as well.

For proving a more general statement we need some preparatory lemmas.

Lemma 5.5. Let $M$ be an R-module. For every $n \geq 1$, let $s_{n} \in R$ and let $\left(s_{n} M\right)_{n \geq 1}$ be an increasing chain of nonzero modules, such that $M=$ $\bigcup_{n \geq 1} s_{n} M$. Then:

(1) $\left(\left(s_{n} M\right)_{\#}\right)_{n \geq 1}$ is an increasing sequence and $M_{\#}=\bigcup_{n \geq 1}\left(s_{n} M\right)_{\#}$,

(2) $\left(\left(s_{n} M\right)^{\#}\right)_{n \geq 1}$ is an increasing sequence and $M^{\#}=\bigcup_{n \geq 1}\left(s_{n} M\right)^{\#}$,

(3) $M^{\#}=\bigcup_{n \geq 1} s_{n} R$, provided that the chain $\left(s_{n} M\right)_{n \geq 1}$ is strictly increasing.

Proof. The fact that the sequences considered in (1) and (2) are increasing follows by Lemma 2.3 .

(1) This immediately follows from the fact that $r \in M_{\#}$ if and only if $M[r] \neq 0$ and that $(s M)[r]=s M \cap M[r]$.

(2) Let $r \in M^{\#}$; that is $r M<M$. Then already $r M<s_{m} M$ for some $m \geq 1$. Hence $r\left(s_{m} M\right) \leq r M<s_{m} M$ and $r \in\left(s_{m} M\right)^{\#} \leq \bigcup_{n \geq 1}\left(s_{n} M\right)^{\#}$. Conversely, by Lemma $2.3,\left(s_{n} M\right)^{\#} \leq M^{\#}$ for every $n$, hence $\bigcup_{n \geq 1}\left(s_{n} M\right)^{\#} \leq$ $M^{\#}$.

(3) If $r \in M^{\#}$ then, as in part (2), $r M<s_{m} M$ for some $m \geq 1$, so $r R<s_{m} R \leq \bigcup_{n \geq 1} s_{n} R$. Conversely, if $r \in \bigcup_{n \geq 1} s_{n} R$, then $r \in s_{m} R$ for some $m \geq 1$. Hence $r M \leq s_{m} M$ and the assumption $s_{m} M<M$ implies $r \in M^{\#}$.

Lemma 5.6. Let $P_{1} \leq P_{2} \leq P_{3} \leq \ldots$ be an ascending chain of prime ideals in $R$ and let $P=\bigcup_{n \geq 1} P_{n}$. If $J$ is an $R$-submodule of $Q$ such that $J \otimes_{R} R_{P_{n}}$ 
is a countably generated $R_{P_{n}}$-module for each $n \geq 1$, then $J \otimes_{R} R_{P}$ is a countably generated $R_{P}$-module.

Proof. Suppose that $J \otimes_{R} R_{P}$ is not countably generated; that is, there is an uncountable regular cardinal $\kappa$ and a strictly ascending chain $\left(x_{\alpha} R_{P}\right)_{\alpha<\kappa}$ such that $J \otimes_{R} R_{P}=\bigcup_{\alpha<\kappa} x_{\alpha} R_{P}$. In particular, $x_{\alpha} x_{\beta}^{-1} \in P=\bigcup_{n \geq 1} P_{n}$ for every $\alpha<\beta<\kappa$. Hence for some $n$, the set

$$
\left\{\alpha<\kappa \mid x_{\alpha} x_{\alpha+1}^{-1} \in P_{n}\right\}
$$

must be of cardinality $\kappa$, or equivalently: unbounded in $\kappa$. For this $n$, the chain $\left(x_{\alpha} R_{P_{n}}\right)_{\alpha<\kappa}$ has a strictly increasing well-ordered cofinal subchain, which implies that

$$
J \otimes_{R} R_{P_{n}}=\bigcup_{\alpha<\kappa} x_{\alpha} R_{P_{n}}
$$

is not countably generated.

Lemma 5.7. Let $M$ be an $R$-module. Assume that $M$ is the union of an ascending chain $0<s_{1} M \leq s_{2} M \leq s_{3} M \leq \ldots$ and all $s_{n} M$ are $\Sigma$-cotorsion. Then the following hold.

(1) The set $\mathcal{S}=\{t M \mid t \in R\}$ is countable and well-ordered,

(2) If $L=M^{\#}$ and $P=M_{\#}$, then every $R_{P}$-submodule $J$ of $Q$ such that $L<J \leq R_{L}$ is countably generated (as an $R_{P}$-module).

Proof. (1) By Corollary 3.5, the sets $\mathcal{S}_{n}=\left\{t s_{n} M \mid t \in R\right\}$ are countable and well-ordered. Therefore, the set $\mathcal{S}=\{t M \mid t \in R\}$ is countable well-ordered as well since $\mathcal{S}=\bigcup_{n \geq 1} \mathcal{S}_{n} \cup\{M\}$.

(2) Denote $L_{n}=\left(s_{n} M\right)^{\#}$ and $P_{n}=\left(s_{n} M\right)_{\#}$ for every $n \geq 1$. By Lemma 5.5 the sequence $\left(L_{n}\right)_{n \geq 1}$ is an increasing sequence and $L=\bigcup_{n \geq 1} L_{n}$. Let $L<J \leq R_{L}$. Then also $L_{n}<J \leq R_{L_{n}}$ for each $n$. Hence $J \otimes_{R} R_{P_{n}}$ is a countably generated $R_{P_{n}}$-module for each $n$ by Lemma 4.2 and consequently $J$ is a countably generated $R_{P}$-module by Lemmas 5.5 and 5.6.

Now we can prove a crucial statement which is a partial converse of Theorem 5.4. Indeed, Theorem 5.4 implies that every $\Sigma$-cotorsion module $M$ is the union of a countable strictly increasing chain of the form $\left(s_{i} M\right)_{i}$ and all the modules $s_{i} M$ are $\Sigma$-cotorsion. Given such a chain $\left(s_{i} M\right)_{i}$ with all the "smaller" modules $s_{i} M$ $\Sigma$-cotorsion, one may ask whether $M$ is necessarily $\Sigma$-cotorsion, too. For a finite chain this is obvious, but for a countable chain this is not trivial and is the contents of the following theorem:

Theorem 5.8. Let $R$ be a valuation domain and $M$ be a nonzero $R$-module. Assume that $M$ is the union of a countable ascending chain $0<s_{1} M \leq$ $s_{2} M \leq s_{3} M \leq \ldots$ and all $s_{n} M$ are $\Sigma$-cotorsion. Then $M$ is $\Sigma$-cotorsion.

Proof. If the chain is finite, then clearly $M$ is $\Sigma$-cotorsion. We can, therefore, assume that $s_{n} M<s_{n+1} M$, for each $n \geq 1$. By Lemma 5.7 the set $\mathcal{S}=$ $\{t M \mid t \in R\}$ is countable well-ordered.

We denote $L=M^{\#}, P=M_{\#}, L_{n}=\left(s_{n} M\right)^{\#}$ and $P_{n}=\left(s_{n} M\right)_{\#}$ for each $n \geq 1$. By Lemmas 2.2 and 5.5, the following hold:

(1) $L \leq P$ and $L_{n} \leq P_{n}$; 
(2) the sequences $\left(L_{n}\right)_{n \geq 1}$ and $\left(P_{n}\right)_{n \geq 1}$ are increasing;

(3) $L=\bigcup_{n \geq 1} L_{n}=\bigcup_{n \geq 1} s_{n} R$ and $P=\bigcup_{n \geq 1} P_{n}$.

Moreover, by Lemma 2.5 (2), we can assume that $P$ is the maximal ideal of $R$.

We first make the following assumption:

$$
L=P \text { and } P_{n}<P, \text { for each } n \geq 1 .
$$

We show that $M$ is $\Sigma$-cotorsion under this assumption by using the characterization of $\Sigma$-cotorsion modules given in Theorem 3.8. We already noticed that $\mathcal{S}=\{t M \mid t \in R\}$ is countable and well-ordered, hence $M$ satisfies condition (1) in that theorem. What remains to prove is that the condition $C C(\kappa, M)$ is satisfied for every uncountable regular cardinal $\kappa$. In other words, we have to prove $C C(\mathfrak{F}, M)$ whenever $\mathfrak{F}=\left(r_{\alpha \beta}\right)_{\alpha<\beta<\kappa}$ is a $\kappa$-factoring system. Note that this is trivial when the chain $\left(r_{0 \gamma}^{-1} R\right)_{\gamma<\kappa}$ is stationary, or, in other words, if there is some $\delta<\kappa$ such that $r_{\alpha \beta}$ is a unit in $R$ whenever $\delta<\alpha<\beta<\kappa$. Let us assume that this is not the case. Then there is a continuous strictly increasing function $f: \kappa \rightarrow \kappa$ such that $r_{f(\alpha) f(\beta)} \in P=\bigcup_{n \geq 1} P_{n}$ whenever $\alpha<\beta<\kappa$. As in the proof of Lemma 5.6, there exists $m \geq 1$ such that the set

$$
E=\left\{\alpha<\kappa \mid r_{f(\alpha) f(\alpha+1)} \in P_{m}\right\}
$$

is unbounded in $\kappa$. When changing $f$ accordingly, we can without loss of generality assume that $E=\kappa$. Note also that $C C(\mathfrak{F}, M)$ holds if and only if $C C\left(\mathfrak{F}^{\prime}, M\right)$ holds where $\mathfrak{F}^{\prime}=\left(r_{f(\alpha) f(\beta)}\right)_{\alpha<\beta<\kappa}$, since $f$ is continuous. Thus, we can pass from $\mathfrak{F}$ to $\mathfrak{F}^{\prime}$ and assume w.l.o.g. that all $r_{\alpha \beta}$ are in $P_{m}$.

We know that for each $n \geq 1, C C\left(\kappa, s_{n} M\right)$ is satisfied, since $s_{n} M$ is $\Sigma$ cotorsion. Thus, for each $n \geq 1$ there is a closed unbounded subset $C_{n} \subseteq \kappa$ with the property that for each limit ordinal $\gamma \in C_{n}$, the descending chain $\left(s_{n} M\left[r_{\alpha \gamma}\right]\right)_{\alpha<\gamma}$ is stationary. Let $C=\bigcap_{n>1} C_{n}$. Then $C$ is still a closed unbounded subset of $\kappa$. Let $j \geq 1$ be such that $P_{m}<s_{j} R$. Such an index $j$ exists since $P_{m}<P$ and $P=L=\bigcup_{n \geq 1} s_{n} R$ by $(\mathbf{I})$. For every limit ordinal $\gamma \in C$ there is $\alpha_{j}<\gamma$ such that

$$
s_{j} M\left[r_{\alpha_{j} \gamma}\right]=s_{j} M\left[r_{\beta \gamma}\right] \text {, for each } \alpha_{j} \leq \beta<\gamma .
$$

We prove that $\alpha_{j}$ is a stationary index for the chain $\left(s_{k} M\left[r_{\alpha \gamma}\right]\right)_{\alpha<\gamma}$, for each $k \geq j$ and thus also for the chain $\left(M\left[r_{\alpha \gamma}\right]\right)_{\alpha<\gamma}$. This will be accomplished in several steps.

First consider the ideal $I_{\gamma}$ of $R$ generated by $r_{\beta \gamma}$ for each $\alpha_{j} \leq \beta<\gamma$. Then

$$
I_{\gamma}=I_{\gamma} s_{k}
$$

for each $k \geq j$. In fact, for each $\beta<\gamma$ we have $r_{\beta \gamma}=r_{\beta, \beta+1} r_{\beta+1, \gamma}$ and $r_{\beta, \beta+1} \in P_{m}<s_{k} R$, for each $k \geq j$. Hence, $r_{\beta \gamma} \in r_{\beta+1, \gamma} s_{k} R$, for each $k \geq j$ and thus $I_{\gamma} \leq I_{\gamma} s_{k}$. This proves (1).

If $r_{\alpha_{j} \gamma} s_{j} M=0$, then by $(*)$ we have that $r_{\beta \gamma} s_{j} M=0$, for each $\alpha_{j} \leq \beta<$ $\gamma$. Hence, by (1), $0=I_{\gamma} s_{j} M=I_{\gamma} M=I_{\gamma} s_{k} M$, for each $k \geq j$. This means that $s_{k} M\left[r_{\alpha_{j} \gamma}\right]=s_{k} M=s_{k} M\left[r_{\beta \gamma}\right]$, for each $\alpha_{j} \leq \beta<\gamma$. 
If $r_{\alpha_{j} \gamma} s_{j} M>0$, then also $r_{\beta \gamma} s_{j} M>0$, for each $\alpha_{j} \leq \beta<\gamma$. Then, by Lemma $1.10(2)$, condition $(*)$ is equivalent to

$$
r_{\alpha_{j} \beta} \notin\left(r_{\beta \gamma} s_{j} M\right)_{\#}, \text { for each } \alpha_{j} \leq \beta<\gamma \text {. }
$$

Condition (a) implies that $\left(r_{\beta \gamma} s_{j} M\right)_{\#}<P_{m}$ for each $\alpha_{j}<\beta<\gamma$, hence $\left(I_{\gamma} s_{j} M\right)_{\#}=\left(I_{\gamma} M\right)_{\#} \leq P_{m}$.

Assume by way of contradiction that $\alpha_{j}$ is not a stationary index for the chain $\left(s_{k} M\left[r_{\alpha \gamma}\right]\right)_{\alpha<\gamma}$, for some $k>j$. Then there exists $\beta>\alpha_{j}$ such that $s_{k} M\left[r_{\alpha_{j} \gamma}\right]>s_{k} M\left[r_{\beta \gamma}\right]$. Since, $r_{\beta \gamma} s_{k} M>0$, Lemma 1.10 (2) implies that

$$
r_{\alpha_{j} \beta} \in\left(r_{\beta \gamma} s_{k} M\right)_{\#} \text {. }
$$

Conditions (a) and (b) imply in particular, that $r_{\beta \gamma} s_{k} M>r_{\beta \gamma} s_{j} M$. So $s_{j} s_{k}^{-1} \in\left(r_{\beta \gamma} s_{k} M\right)^{\#}$. By assumption $s_{k} M$ is $\Sigma$-cotorsion, so the same holds for $r_{\beta \gamma} s_{k} M$ by Theorem 3.9. Thus, $\left(r_{\beta \gamma} s_{k} M\right)^{\#} \leq\left(r_{\beta \gamma} s_{k} M\right)_{\#}$ by Proposition 2.1 and Lemma 2.2. Now $r_{\beta \gamma} s_{k} M \leq I_{\gamma} s_{k} M=I_{\gamma} M$. Hence, $\left(r_{\beta \gamma} s_{k} M\right)^{\#} \leq\left(r_{\beta \gamma} s_{k} M\right)_{\#} \leq\left(I_{\gamma} M\right)_{\#} \leq P_{m}$. Thus, $s_{j} s_{k}^{-1} \in P_{m}$ and $s_{j} \in s_{k} P_{m} \leq P_{m}$, contradicting the assumption $P_{m}<s_{j} R$. We have, therefore, shown that $C C(\kappa, M)$ is satisfied and we conclude that $M$ is $\Sigma$ cotorsion.

Now assume that hypothesis (I) does not hold and suppose, by way of contradiction, that $M$ is not $\Sigma$-cotorsion. Then, for each $m \geq 1, M / s_{m} M$ is not $\Sigma$-cotorsion. Note that $L_{m} \leq \sqrt{\operatorname{Ann}_{R}\left(M / s_{m} M\right)}$ by Lemmas 2.4 and 5.3. Hence, by Proposition 2.6, there exists an $R$-submodule $J_{m}$ of $Q$ and a set $Y_{m}$ such that $L_{m}<J_{m} \leq R_{L_{m}}$ and $\operatorname{Ext}_{R}^{1}\left(J_{m},\left(M / s_{m} M\right)^{\left(Y_{m}\right)}\right) \neq 0$. Let $Y=\bigcup_{m>1} Y_{m}$. Since by hypothesis $s_{m} M$ is $\Sigma$-cotorsion, we see that $\operatorname{Ext}_{R}^{1}\left(J_{m}, M^{(\bar{Y})}\right) \neq 0$ for each $m \geq 1$. Note that, for each $m \geq 1, J_{m}$ is not a countably generated $R$-module, since otherwise $\operatorname{Ext}_{R}^{1}\left(J_{m}, M^{(Y)}\right)=0$ by Proposition 2.1 .

Fix $m \geq 1$. Lemma 5.6 implies that there exists $n \geq 1$ such that $J_{m} \otimes_{R}$ $R_{P_{n}}$ is not a countably generated $R_{P_{n}}$-module. So there is an uncountable regular cardinal $\kappa$ and a strictly ascending chain $\left(x_{\alpha} R_{P_{n}}\right)_{\alpha<\kappa}$ such that $J_{m} \otimes_{R} R_{P_{n}}=\bigcup x_{\alpha} R_{P_{n}}$. Consider $J_{m} \otimes_{R} R_{P_{m}}$; since $L_{m} \leq P_{m}$ we have $L_{m}<J_{m} \otimes_{R} R_{P_{m}} \leq R_{L_{m}}$ and, by Lemma 4.2 used for $s_{m} M$, we infer that $J_{m} \otimes_{R} R_{P_{m}}$ is a countably generated $R_{P_{m}}$-module. Hence in particular $n>m$. The chain $\left(x_{\alpha} R_{P_{m}}\right)_{\alpha<\kappa}$ must be constant from some point on and consequently $J_{m} \otimes_{R} R_{P_{m}}=b R_{P_{m}}$ for some $b \in Q$. Now, if $L_{n} \leq P_{m}$, then $L_{n}<b^{-1} J_{m} \leq R_{L_{n}}$ and $b^{-1} J_{m} \otimes_{R} R_{P_{n}}$ would be an uncountably generated $R_{P_{n}}$-module. But this contradicts Lemma 4.2 and the assumption that $s_{n} M$ was a $\Sigma$-cotorsion module. So, for each $m \geq 1$ there is $n>m$ such that $L_{n}>P_{m}$. This implies that $P_{m}<P$ for each $m \geq 1$ and $L=P$. But we have seen above that in this case $M$ is $\Sigma$-cotorsion. This completes the proof.

Strictly speaking, Theorems 5.4 and 5.8 are not really converses of each other. We conclude the paper by combining the two theorems to a corollary which makes precise, in which way a $\Sigma$-cotorsion module is reconstructed from divisible $\Sigma$-cotorsion modules over factor domains. 
Corollary 5.9. Let $R$ be a valuation domain and $M$ be an $R$-module. Then the following are equivalent:

(1) $M$ is $\Sigma$-cotorsion.

(2) The set $\mathcal{S}=\{I M \mid I \leq R\}$ is countable, well-ordered by inclusion, and $I M / J M$ is $\Sigma$-cotorsion (and divisible over a suitable valuation factor domain) whenever $I M$ is an immediate successor of $J M$ in $\mathcal{S}$.

Proof. The implication (1) $\Longrightarrow(2)$ is a direct consequence of Theorem 5.4. Suppose that (2) holds. As $\mathcal{S}$ is well-ordered, we can prove that $I M$ is $\Sigma$ cotorsion for each ideal $I$ of $R$ by transfinite induction. For $I M=0$, this is clear. Otherwise, put $N=\bigcup_{s \in(I M) \#} s I M$. If $N=I M$, then $I M$ is a union of a (countable) chain $0<s_{1} I M<s_{2} I M<s_{3} I M<\ldots$, so we can use Theorem 5.8 to conclude that $I M$ is $\Sigma$-cotorsion. If $N<I M$, then clearly $I M$ is an immediate order-successor of $N$ in $\mathcal{S}$. Therefore, $I M / N$ is $\Sigma$ cotorsion by assumption, and so is $I M$ itself using the inductive hypothesis. Hence (1) follows by putting $I=R$.

\section{REFERENCES}

[1] L. Angeleri Hügel, S. Bazzoni and D. Herbera, A solution to the Baer splitting problem, Trans. Am. Math. Soc. 360 (2008), no.5, 2409-2421.

[2] H. Bass, Finitistic dimension and a homological generalization of semiprimary rings, Trans. Amer. Math. Soc. 95(1960), 466-488.

[3] S. Bazzoni and D. Herbera, One dimensional tilting modules are of finite type, Algebr. Represent. Theory, 11 (2008), no.1, 43-61.

[4] S. Bazzoni and D. Herbera, Cotorsion pairs generated by modules of bounded projective dimension, to appear in Israel J. Math.

[5] S. Bazzoni and L. Salce, An Independence Result on Cotorsion Theories over Valuation Domains, J. Algebra 243 (2001), 294-320.

[6] R. Camps, A. Facchini, The Prüfer rings that are endomorphism rings of artinian modules, Comm. Algebra 22 (1994), 3133-3157.

[7] H. Cartan and S. Eilenberg, Homological Algebra, Princeton University Press, 1956.

[8] C. C. Chang and H. J. Keisler, Model Theory, North-Holland, Amsterdam, 1973.

[9] P. Eklof and A. Mekkler, Almost-free Modules, North-Holland Math. Library, Revised Edition, Elsevier 2002.

[10] I. Emmanouil, Mittag-Leffler condition and the vanishing of $\lim ^{1}$, Topology 35 (1996), no. $1,267-271$.

[11] A. Facchini and G. Puninski, $\Sigma$-pure-injective modules over a serial ring, Abelian groups and modules, Kluwee Acad. Publishers, Dodrecht, (1995), 145-162.

[12] L. Fuchs and L. Salce, Modules over Non-Noetherian Domains, Mathematical Surveys and Monographs, American Mathematical Society, Providence, 2001.

[13] P. Guil-Asensio and I. Herzog, Model-theoretic aspects of $\Sigma$-cotorsion modules, Ann. Pure Appl. Logic 146 (2007), 1-12.

[14] L. Gruson and C. U. Jensen, Deux applications de la notion de L-dimension, C. R. Acad. Sci. Paris, Sér. I.Math. 282 (1976), 23-24.

[15] A. Grothendieck, Éléments de géométrie algébrique. III. Étude cohomologique des faisceaux cohérents, Inst. Hautes Études Sci. Publ. Math. No. $11,1961$.

[16] D. K. Harrison, Infinite abelian groups and homological methods, Ann. of Math. 69 (1959), 366-391.

[17] T. Jech, Set theory, Pure and Applied Mathematics, Academic Press (Harcourt Brace Jovanovich, Publishers), New York-London, 1978. 
[18] C. U. Jensen, Homological dimension of rings with countably generated ideals, Math. Scand. 18 (1966), 97-105.

[19] C. U. Jensen, Les foncteurs dérivé del lim et leur Applications en Théorie des Modules, Lecture Notes in Mathematics, Vol. 254, Springer Verlag, Berlin-New York 1972.

[20] B. Mitchell, The cohomological dimension of a directed set, Canad. J. Math. 25 (1973), 233-238.

[21] E. Monari-Martinez, On $\Sigma$-pure-injective modules over valuation domains, Arch. Math. 46 (1986), 26-32.

[22] M. Prest and G. Puninski, $\Sigma$-pure-injective modules over a commutative Prüfer ring, Comm. Algebra 27 (2) (1999), 961-971.

[23] B. Osofsky, A Remark on colimits, XXVII (3) (1975), 496-499.

[24] J. Štovíček and J. Trlifaj, All tilting modules are of countable type, Bull. Lond. Math. Soc. 39 (2007), 121-132.

[25] C. A. Weibel, An introduction to homological algebra, Cambridge Studies in Advanced Mathematics, 38. Cambridge University Press, Cambridge, 1994.

[26] M. Ziegler, Model theory of modules, Ann. Pure Appl. Logic. 26 (1984), 149-213.

[27] W. Zimmermann, Rein-injektive direkte Summen von Moduln, Comm. in Alg.5 (1977), 1083-1117.

Dipartimento di Matematica Pura e Applicata, Universitá di Padova, Via Belzoni 7, 35131 PAdOVA

E-mail address: bazzoni@math.unipd.it

INSTITUTT FOR MATEMATISKE FAG, NORGES TEKNISK-NATURVITENSKAPELIGE UNIVERSitet, N-7491 Trondheim, Norway

E-mail address: stovicek@math.ntnu.no 